OPEN ACCESS

Edited by:

Junli $X u$,

University College Dublin, Ireland

Reviewed by:

Zhaoli Zhang,

Yangzhou University, China Zhoui Wang

Northwest $A$ and F University, China

${ }^{*}$ Correspondence:

Haile Ma

mhl@ujs.edu.cn

Specialty section:

This article was submitted to

Nutrition and Food Science

Technology,

a section of the journal

Frontiers in Nutrition

Received: 18 June 2021

Accepted: 29 September 2021

Published: 03 November 2021

Citation:

Luo X, Dong Y, Gu C, Zhang X and Ma H (2021) Processing Technologies

for Bee Products: An Overview of

Recent Developments and

Perspectives. Front. Nutr. 8:727181

doi: 10.3389/fnut.2021.727181

\section{Processing Technologies for Bee Products: An Overview of Recent Developments and Perspectives}

\author{
Xuan Luo, Yating Dong, Chen Gu, Xueli Zhang and Haile Ma* \\ School of Food and Biological Engineering, Jiangsu University, Zhenjiang, China
}

Increased demand for a more balanced, healthy, and safe diet has accelerated studies on natural bee products (including honey, bee bread, bee collected pollen royal jelly, propolis, beeswax, and bee venom) over the past decade. Advanced food processing techniques, such as ultrasonication and microwave and infrared $(\mathrm{IR})$ irradiation, either has gained popularity as alternatives or combined with conventional processing techniques for diverse applications in apiculture products at laboratory or industrial scale. The processing techniques used for each bee products have comprehensively summarized in this review, including drying (traditional drying, infrared drying, microwave-assisted traditional drying or vacuum drying, and low temperature high velocity-assisted fluidized bed drying), storage, extraction, isolation, and identification; the assessment methods related to the quality control of bee products are also fully mentioned. The different processing techniques applied in bee products aim to provide more healthy active ingredients largely and effectively. Furthermore, improved the product quality with a shorter processing time and reduced operational cost are achieved using conventional or emerging processing techniques. This review will increase the positive ratings of the combined new processing techniques according to the needs of the bee products. The importance of the models for process optimization on a large scale is also emphasized in the future.

Keywords: bee products, processing techniques, drying, wall-breaking, extraction, identification, quality control

\section{INTRODUCTION}

Natural products and preparations for food and nutritional supplement or dietetic purposes have been used in folk medicine for several years (1). Apiculture products have long been used for phytotherapy and diet because of their positive effects on health $(2,3)$.

The history of bee products and their therapeutic effects date back to the ancient times. Healing properties of the bee products are recorded in many religious texts, including the Bible, Vedas, and Quran $(4,5)$. Bee collected pollen (BCP) and bee bread (BB) are used as food supplements for humans (6), even the Greeks believed that BCP and honey were the food of kings, which can give them the youthfulness and life. Nowadays, bee products [honey, royal jelly (RJ), propolis, beeswax, bee venom (BV), bee collected pollen (BCP), and bee bread (BB)] as natural medicines are gaining prominence for the full of bioactive compounds that are associated with their high bioactive molecule content (7), beneficial health properties $(4,8)$, including powerful healing properties. Thus, there is an increasing demand for these natural bee products currently. 
The biologically active components of bee products include carbohydrates (9), proteins, peptides (10), lipids (11), vitamins $(12,13)$, minerals (14), polyphenols, flavonoids (15), terpenoids $(16,17)$, and a small amount of other compounds. The nutritional value of their chemical components certainly improves health and body functioning (12). Multiple studies also shown that for the active compounds contained in the bee products made them possess excellent antioxidant (17), antimicrobial $(18,19)$, antiinflammatory, immunomodulatory (20), antiproliferative (21), anticarcinogenic, antitumor (22), and antiallergic properties. Served in the form of tablets, capsules, powders, granules, candy bars, oral liquids, and tonics or as received, bee products are particularly recommended as dietary supplements for human consumption $(14,23)$.

China is a large global producer of apiculture commodities, to fully develop these resources, maintain quality at the desired level, achieve maximum benefits and nutritional values, and produce each type of bee product as a dietary supplement for human beings, more effective processing techniques are badly needed to produce more nutritional and high-quality apiculture products.

Many external factors, including, temperature, humidity affected the physicochemical properties of bee products, even the processing treatments, such as ultrasonic assisted extraction, microwave-assisted drying (MWD), and analytical techniques $(24,25)$ can strongly influence the content of the active ingredients and their bioactivity. An integrated approach would be useful and efficient for the preservation of bioactivity ingredients during the production and processing stages. Physical processing of bee products focus on changing the moisture content (26), enhancing the extraction $(27,28)$, modifying the external structures (29).

In previous, several reviews have focused on the bioactivity and therapeutic properties $(18,30-33)$ of bee products; bee products active ingredients from different botanical origins and regions (34, 35); extraction (11) and analytical techniques (36) have been used to obtain, purify, identify, characterize, and/or quantify bioactive compounds (37). However, few reviews have comprehensively summarized the processing technologies applied in the bee products.

Thus, this review aims to present recent developments in processing techniques applied in bee products, which will strengthen the existing knowledge regarding bee product processing techniques and proposing potential combine processing strategies for better processing the bee products.

\section{BEE PRODUCTS}

Honeybee-derived products are used as traditional complementary medicines worldwide, especially in oriental countries (38). Bee products can be divided into three categories: (1) bee collection and brewing products, such as propolis, honey and $\mathrm{BCP}, \mathrm{BB}$; (2) Bee secretions, such as RJ, beeswax, and BV; and (3) bee ecological bodies and hives, such as bee larvae, bee corpses, and old beehives (39) (Figure 1). Hive products and their apitherapy have a long history dates back to the ancient times, which have been used in phytotherapy and diet for their powerful healing properties (6). Over the past 5 years, several research papers have been published focus on the beneficial compounds, data from the web of science were shown in Figure 2.

\section{Bee Collection and Brewing Products Honey}

Reports of ancient populations using honey for both nutritional and medicinal properties can be traced back to nearly 5,500 years ago $(41,42)$. Honey as a natural product produced from the nectar of flowers by honeybees (43). Because of its high nutritive components, it has been traditionally used to treat wounds and diseases of the gut, including gastric ulcers, used as a remedy for sore throat, cough, hepatitis, thirst, earaches, hiccups, fatigue, worm infections, tuberculosis, dizziness, constipation, piles, eczema (44-46).

Around 300 types of honey have been identified (47). The main component of honey is carbohydrate, which contributes 95-97\% of its dry weight (48), predominantly fructose and glucose $(40,49,50)$. Furthermore, honey contains proteins (0.5\%), vitamins, more than 20 amino acids, minerals (0.04$0.2 \%$ ), organic acids (0.57\%), enzymes (sucrose, diastase or amylase; CAT; invertase, $\alpha$-glucosidase; and glucose oxidase), and solid particles derived from the honey collection (51). Flavonoids and polyphenols are the two main bioactive (antioxidants) molecules present in honey $(41,52,53)$.

\section{Bee Pollen and Bee Bread}

Both $\mathrm{BCP}$ and $\mathrm{BB}$ are rich in nutritional components like sugars, proteins, fatty acids (including $\omega-3$ and $\omega-6$ fatty acids), essential amino acids, vitamins, and even macro-and microelements (54).

Global production of BCP is $\sim 1,500$ tons per year. The largest producers are China, Australia, and Argentina (55). As a wellknown functional food, the main primary constituents of the BCP are carbohydrates $(12,56,57)$, and water, which are rich in protein $(58,59)$, lipids $(57,60-62)$, dietary fiber, and mineral contents, as well as vitamins and antioxidants (63).

BCP is the basic food for worker bees for RJ production or directly as food (larval queen and worker larvae) because of the large amount of proteins and lipids in it (64-66). The use of BCP for human nutrition on a larger scale began only after the Second World War $(67,68)$.

$\mathrm{BB}$ is a kind of hive product of lactic acid fermentation of BCP, which is unexplored by many beekeepers (52).

BCP are partly destroyed after fermentation, higher nutritional value and bioavailability it possess make it better digestibility than BCP (69). BB contains a few proteins and fats but an abundance of easily assimilated carbohydrates, free amino acids, and lactic acid. The conversion of BCP to BB is a result of biochemical changes, which are mediated by microbial metabolism, principal lactic acid fermentation by bacteria and yeasts (33).

\section{Propolis}

Propolis (bee glue) is a sticky resinous material that released from bud exudates, flowers, and leaves modified by bee secretions 
A

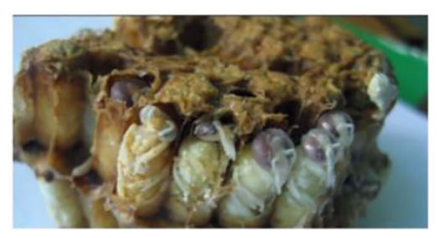

Honeybee pupae

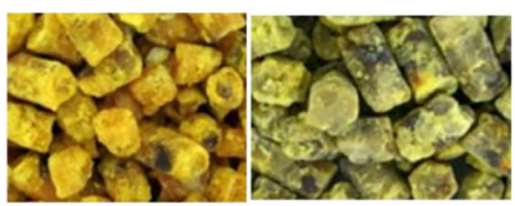

Beebread

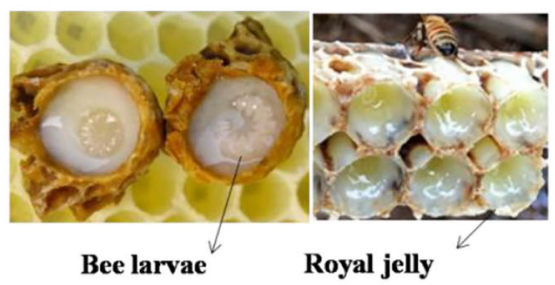

Bee larvae Royal jelly

B

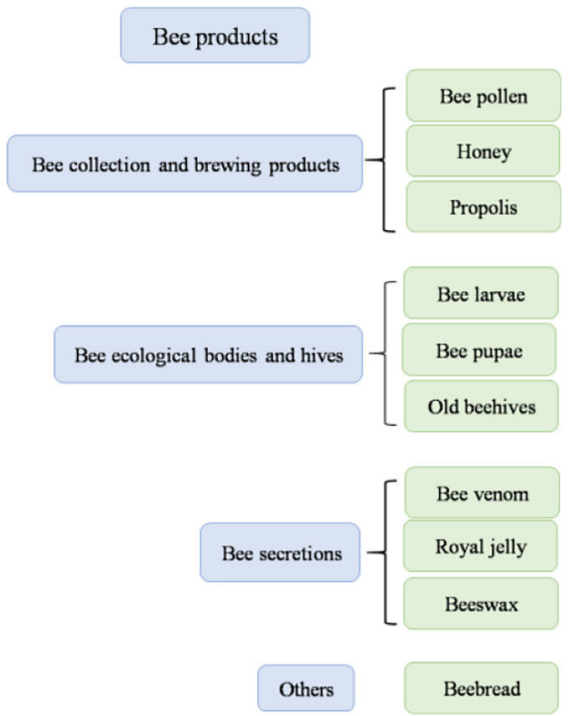

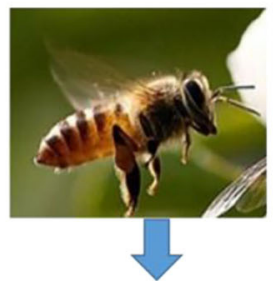
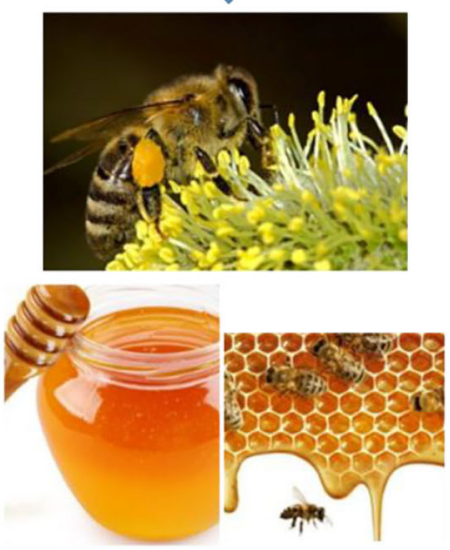

Honey

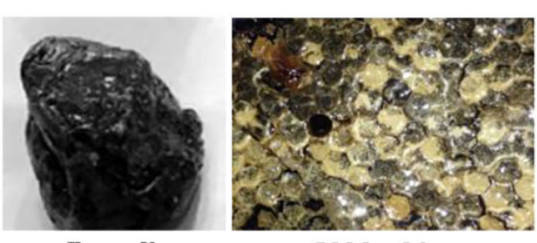

Propolis

Old beehives

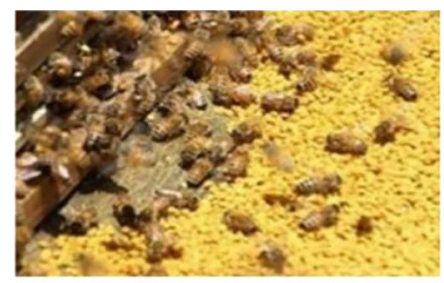

Pollen

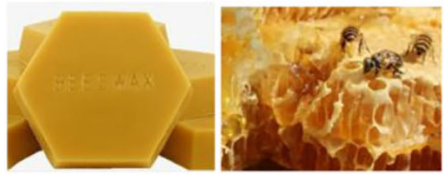

Beeswax
Main compounds

Carbohydrate, protein, lipid, Polyphenols and flavonoid

Carbohydrate, flavonoid

Polyphenols and flavonoid

Proteins, lipids, sugars

Peptide and protein

Carbohydrate, protein, lipid Polyphenols and flavonoid

Protein, lipid, Polyphenols and flavonoid, hydrocarbons

Carbohydrate, protein, lipid, Polyphenols and flavonoid
Bioactivity

Antibiotic, Anti-atherogenic, Anti-anemia, Antitussives, Diuretic, Digestive, Cardioprotective, Hepatoprotective, Kidney function Immunomodulating, Uleer healing etc.

Antioxidant, Antibacterial, Wound dressing, anti-inflammatory, anti-ulcer, antitumour, remedy for cough, sore throat, and caraches etc.

Antibacterial, antioxidant, antiviral, anti-inflammatory, antitumoral, anti proliferative, anti hyperglicemic, antifungal, dental action, antiprotozoan, etc.

\section{Anti-tumor, anti-metastasis, alleviated depression, inhibits}

development of atopic dermatitis-like skin lesions, etc.

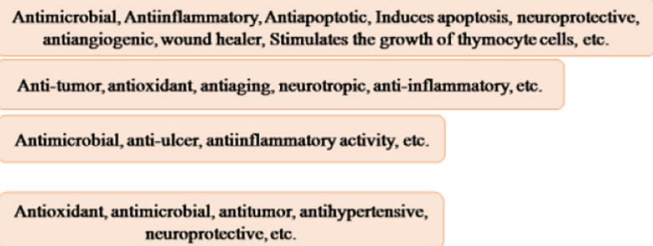

FIGURE 1 | Bee products (A) and their bioactivities (B). Cite from Pasupuleti et al. (40).

(enzymes) and wax (70,71), which are present in bee hives and used by honeybees as a cementing material to close open spaces and cracks occurring in their hives (72). Propolis has been utilized as a helpful drug and nourishment for overall well-being since $300 \mathrm{BCE}(73,74)$. In 1908, the first scientific work was published about the propolis chemical properties and composition (73).
The composition of propolis varies depending on the geographical, vegetation, and ecological region, and even the time and method of the collection $(75,76)$. In general, propolis is composed of $45-55 \%$ vegetable balms and resins (flavonoids, phenolic acids, and esters), 8-35\% wax, 5-10\% essential oils and aromatics (including pinene, viridiflorol, eudesmol, and tricosane), 5\% pollen, 5\% fatty acids, and 


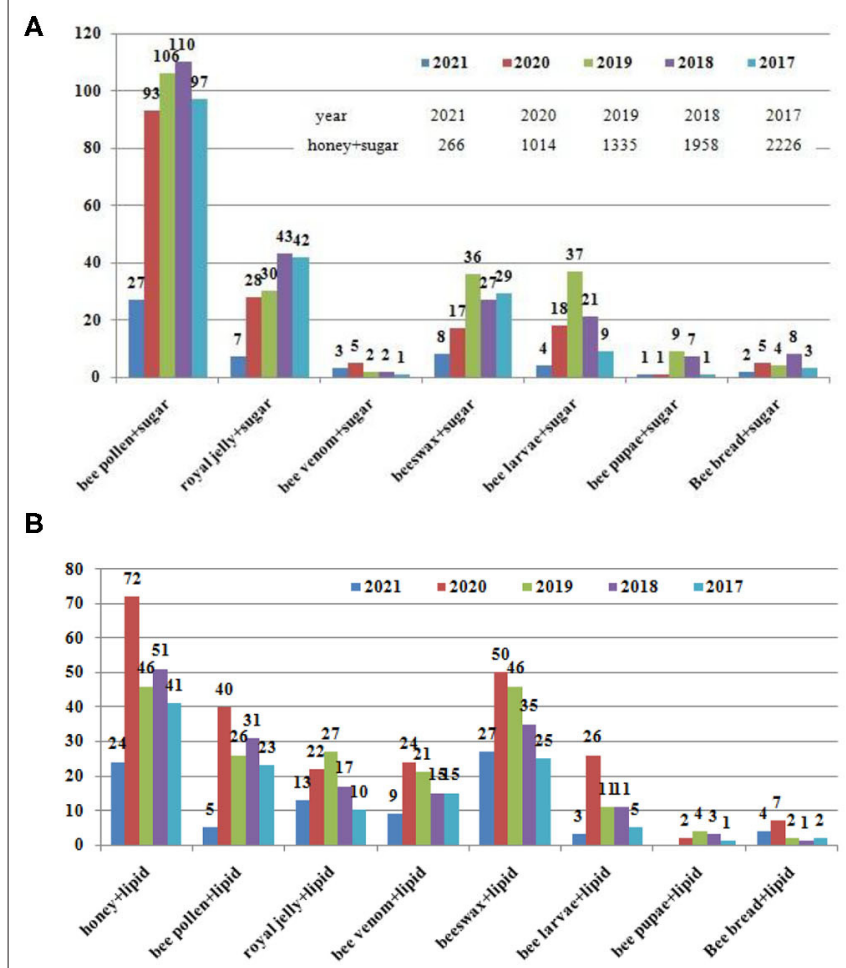

C

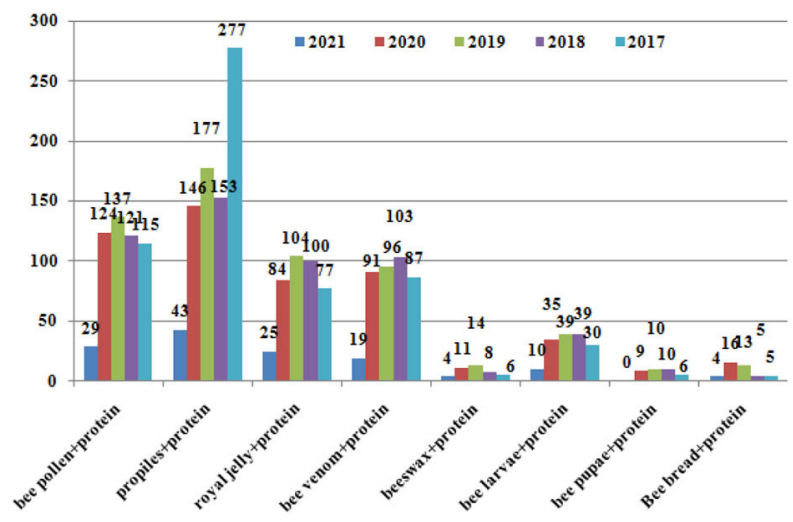

D

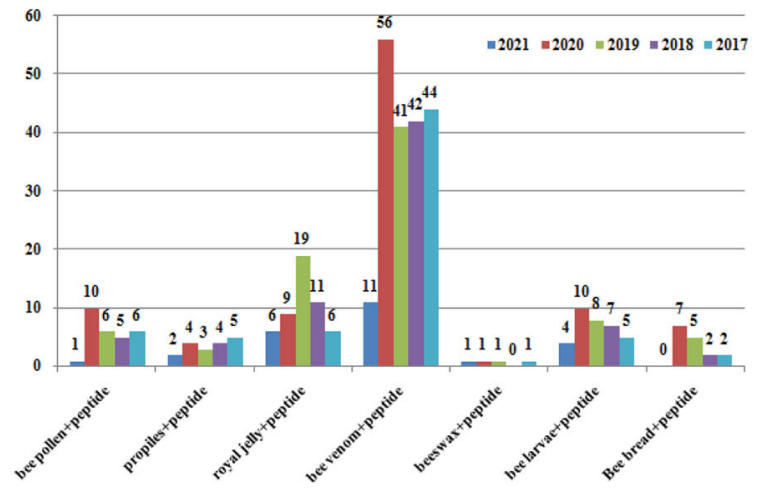

FIGURE 2 | Papers related to bee products published in the recent 5 years (2017-2021). Data from the Web of Science on 4.25. (A) Sugar, (B) lipid, (C) protein, and (D) peptide.
5\% other organic compounds, and minerals (77). Flavonoids, aromatic acids, chlorogenic acids, diterpenic acids, phenolic compounds and their derivatives, fatty acids, prenylated benzophenones, prenylated phenylpropanoid acids, and other secondary metabolites appear to be the principal components responsible for the biological activities of propolis $(21,78)$.

\section{Bee Secretions}

RJ

$\mathrm{RJ}$ is produced by nurse bees to feed young worker larvae during the first 3 days and the entire life of queen bees (79-81). RJ is known as a "superfood," and considered as one of the most appreciated and valued natural products that has been primarily used in traditional medicines, health food, and cosmetics (82). There is a long history about the RJ production techniques. In 1921, Sherlock Holmes simply use a syringe to suck the RJ out of a naturally queen cell. By the $1950 \mathrm{~s}$, the small-scale production and sale of RJ was started by the Mexican, French, and Italian beekeepers (83). China began producing RJ using the method reported in a French literature in 1957 (84).

China is the largest producer and exporter of RJ worldwide, with more than 4,000 tons production annually $90 \%$ of the total RJ production are exported to Japan, Europe, and the United States and more than a $\$ 2.5$ billion market $(84,85)$. Fresh RJ contains $(60-70 \% \mathrm{w} / \mathrm{w})$ water. The dry matter of the RJ is composed of protein $(27-41 \%)$, carbohydrates $(\sim 30 \%)$, lipids $(8-19 \%)$, minerals, some vitamins, and trace elements $(13,58,86-88)$.

\section{Beeswax}

Beeswax is a natural biological polymer (89) isolated from bee secretions, and its chemical composition varies from geographical zones and bee species, although its concentrations may vary depending on the honeybee species and geographical origin. In $150 \mathrm{BC}$, beeswax was one of the components in cosmetic cream created by ancient physician (90), and it also played an important role in ancient and traditional Indian medicine (91). Beeswax are primarily composed of esters, fatty acids, alcohols and hydrocarbons, linear wax monoesters, free fatty alcohols, hydroxy monoesters derived from palmitic, oleic acids, 15-hydroxypalmitic, and complex wax esters containing 15-hydroxypalmitic acid and diols (92). Beeswax are multicomponent solid mixture at room temperature (93), and used as an additive in the food industry, cosmetics, and candles (16). Beeswax is a natural biological polymer (94), which is used as a thickener, drug carrier, binder, and a release retardant in pharmaceutical preparations (95).

\section{BV}

$\mathrm{BV}$ is a discovery dates back to ancient Egypt more than 6,000 years (96). As a therapeutic modality, BV has been used since the second century BC in Eastern Asia (97). BV is a complex natural mixture produced by A. mellifera, an European honeybee (98, 99). It has been widely used as a traditional medicine for treating various diseases (8). It is known to contain peptides [mast cell degranulating (MCD) peptide, melittin, apamin, and adolapin]; 
enzymes (phospholipase A2 (PLA2) and hyaluronidase); amino acids and volatile compounds $(10,100)$.

\section{Bee Ecological Bodies and Hives \\ Bee Larvae and Pupae}

The life cycle of the honeybee has four major stages: egg, larva, pupa, and adult. The larval stage usually lasts $\sim 6$ days (101). As early as $1200 \mathrm{BC}$, the ancient book "Erya" recorded the eating of bees. The silk book "Fifty-two Cases" an ancient medical prescription unearthed in Mawangdui, Changsha, contains a prescription for treating diseases with bee embryos and bees (39). Honeybee larva was first recorded in the earliest extant classic on pharmacology for traditional Chinese medicine, "Shen nong ben cao jing" (102). In China, honeybee larvae have been used to treat and prevent various diseases for more than 1,000 years. Previous study reported that lyophilized powder of honeybee larvae could alleviate tinnitus-associated depression (103).

The protein and fat content of A. mellifera (larvae and pupae) were $42 \pm 0.4-50 \pm 1.6$ and $19 \pm 1.8-21 \pm 1.5$, respectively, and the minerals were $3 \pm 1.3-4 \pm 1.6$ (104). As the larvae progressed to the imago stage, carbohydrate and fat contents decreased from 46.1 and $14.5 \%$ to 30.6 and $6.9 \%$, whereas protein content increased from 35.3 to $51 \%$. Ramos-Elorduy reported the protein content for pupae and bee larvae were 42 and $49 \%$ (dry weight), respectively (105). The pupae and larvae of the honeybee become an especially good source of essential amino acids [16.45/100 g of honey bee larvae powder (HLP)] and fatty acids (106). In addition, honey bee larvae powder extract (HLE) is full of total phenolic content $(51.44 \mathrm{mg} \mathrm{GAE} / \mathrm{g}$ ) and total flavonoid content (2.47 mg RE/g) (22). High antioxidant amino acid residues can be obtained from queen bee larvae $(\mathrm{QBL})$ using simple processing methods (enzymatic hydrolysis and membrane isolation) (107).

Honeybee larvae could also use as potent clinical medicines or functional food for tumor therapy (22) and an adjunctive therapy for the management of atopic dermatitis (AD) (108).

\section{Summary}

- The content of the nutritional components in the three kinds of the bee products are different;

- Carbohydrate are mostly studied in the BCP; Lipids are most studied in the honey, beeswax and BCP; Protein are most studied in the propolis, BCP, RJ, BV; the peptide are most studied in the BV;

- The carbohydrates are the most content in the honey, contributes $95-97 \%$ of its dry weight; Fresh RJ contains (60$70 \% \mathrm{w} / \mathrm{w}$ ) water. The dry matter of the RJ is composed of protein (27-41\%), carbohydrates ( 30\%), lipids (8-19\%); peptides are the main components in BV, $\sim 48-50 \%$ of dry venom were the small proteins and peptides; Propolis is composed of $45-55 \%$ vegetable balms and resins, $8-35 \%$ wax, $5-10 \%$ essential oils and aromatics, $5 \%$ pollen, $5 \%$ fatty acids, and $5 \%$ other organic compounds, and minerals; BCP contains about $15-60 \%$ carbohydrates, total lipid content of the $\mathrm{BCP}$ varying from 1 to $13 \%$ from different botanical species, proteins are the second largest amount of nutritional component in $\mathrm{BCP}$ and fulfill the nutritional requirements of a honeybee. The content in the larvae are different according to the stages.

All the bee products are potent clinical medicines or functional food for human being.

\section{STORAGE OF THE BEE PRODUCTS}

Owing to the high moisture level in the $\mathrm{BCP}$ and $\mathrm{BB}$, the process of dehydration is essential since this moisture causes rapid fermentation and deterioration (109). The vitamin contents of pollen can be altered by processing and storage $(61,110)$. The quality of the product for consumption, from the monitoring of its production, preparation, and storage should be clarified to ensure the beneficial actions of the bee products.

\section{Bee Pollen and Bee Bread}

For fresh BCP with high moisture levels, storage conditions can affect much of the nutrients it contains. The BCP is dried (111) or pulverized (112), freeze-dried $(9,62)$, treated with vacuum to remove the impurities, then packed in glass bottles and stored at $-18^{\circ} \mathrm{C}(9,111,113-115),-20^{\circ} \mathrm{C}(62,116,117), 4^{\circ} \mathrm{C}(36,112$, 118 ) or stored in sterilized containers or bags and stored at $4^{\circ} \mathrm{C}$ $(119,120)$, in the dark at room temperature $\left( \pm 20^{\circ} \mathrm{C}\right)(121)$. Some researchers also homogenized the $\mathrm{BCP}$ samples and stored them under argon at $-24^{\circ} \mathrm{C}(122)$. P. mirabilis was found after 1- and 2 -year vacuum cold storage $\left(0-4^{\circ} \mathrm{C}\right)$ in dried flower BCP (123). It is possible to infer that it is better to consume $\mathrm{BCP}$ frozen at $-20^{\circ} \mathrm{C}$ compared with that dried in an electric oven (124).

Studies have shown that pure dried BB, fresh $\mathrm{BCP}$ and all conserved mixtures with dried $\mathrm{BB}$, fresh $\mathrm{BCP}$ can be stored in a refrigerator at $4-5^{\circ} \mathrm{C}$ for 2 years and at room temperature for 1 year $(34,125)$ without any marked changes in the product quality. Other researchers have also chosen to store bee bread at a lower temperature. Usually, samples are put in a freezer and conserved at $-12^{\circ} \mathrm{C}(126)$. After lyophilization and stored at $-20^{\circ} \mathrm{C}(30,127)$.

\section{RJ and Honey}

Refrigeration and freezing can delay and effectively reduce the chemical changes in RJ during storage, and thus escape the effect of the heat, light, and air. Regarding the shelf-life, Donadieu (128) recommended that the optimal conservation temperature is 0 $5^{\circ} \mathrm{C}$ for fresh RJ preservation for 1 year, whereas the lyophilized RJ can store at room temperature for several years without deterioration (129). A recent study from the MARIA CRISTINA MESSIA who determined the Furosine of the freezed drying RJ during different days and temperature, and pointed out that the lyophilized RJ was more prone to Maillard reaction than fresh RJ for the furosine values risen during the days passing by, even 18 months later. Researchers also pointed that the storage temperature of $4^{\circ} \mathrm{C}$ is appropriate for raw $\mathrm{RJ}(130)$.

The storage temperature was $4^{\circ} \mathrm{C}$, the shelf life of the fresh RJ was 6 months, while for lyophilized RJ, or lyophilized RJ in honey (humidity $<18 \%$ ), and the Apilac pills (lactose-glucose-freezedried $\mathrm{RJ}$ ), the time would be 1 year, 2 year at room temperature, and at $4-8^{\circ} \mathrm{C}$ for 2 years, the shelf lives of fresh RJ and lyophilized 


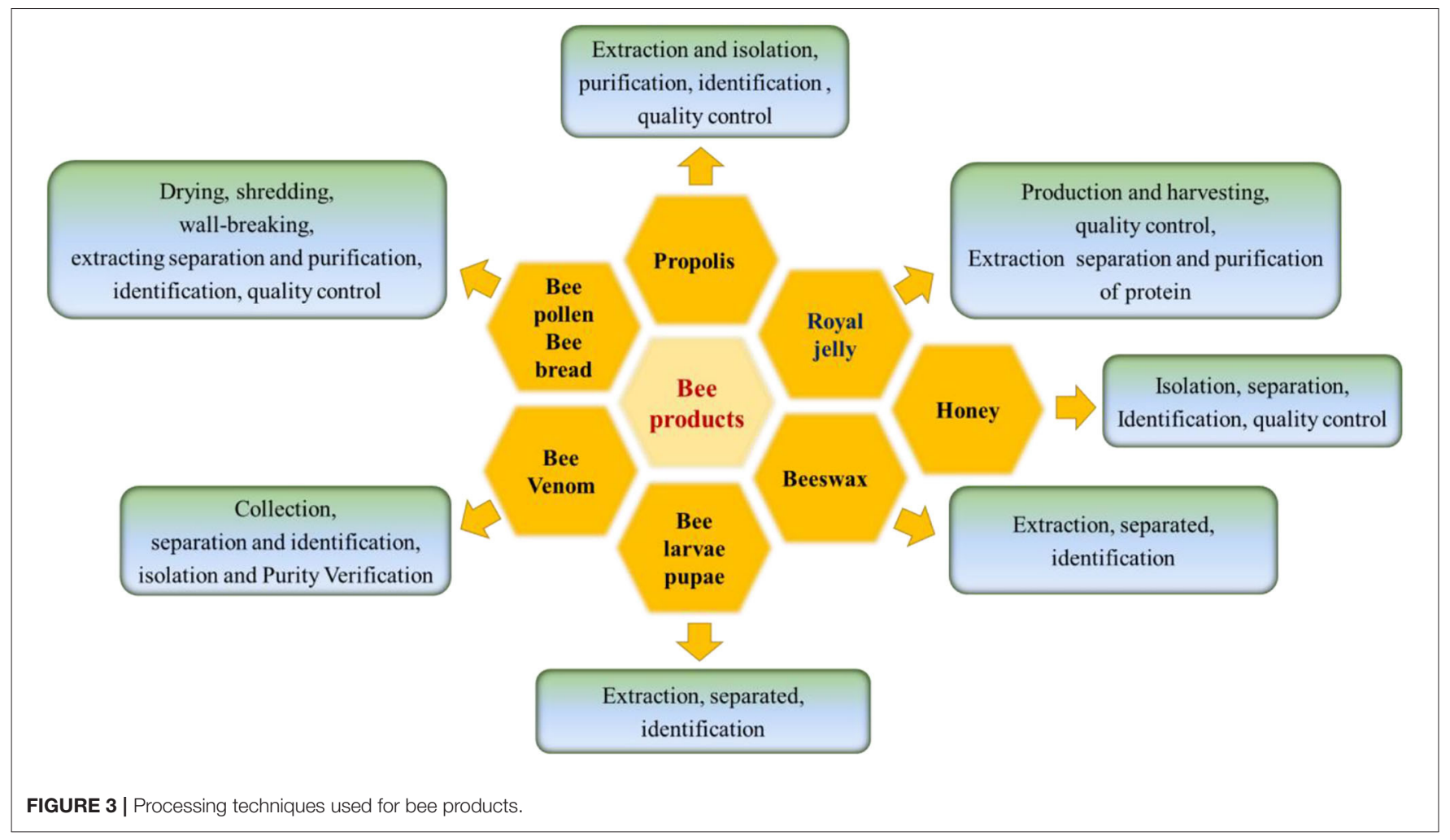

RJ were 2 and $>2$ years, the temperature was set at $<-18^{\circ} \mathrm{C}$, respectively (131). BCP, propolis, honey, and $\mathrm{BB}$ were stored at $6^{\circ} \mathrm{C}$ for a maximum of 4 weeks, while for RJ was stored at $-18^{\circ} \mathrm{C}$ $(52,132)$. The collected honey samples can be stored at $4^{\circ} \mathrm{C}$ $(133,134)$, or $20^{\circ} \mathrm{C}(135)$.

\section{Propolis}

Raw propolis was stored at $4^{\circ} \mathrm{C}$ (136). To ensure the propolis quality, the samples were harvested using a plastic propolis trap and stored in the dark at $-20^{\circ} \mathrm{C}(\sim 30$ days) (15), or even grounded in a marble mortar at $-30^{\circ} \mathrm{C}(137)$.

\section{Summary}

- The storage condition is different according to the bee products;

- Fresh BCP and BB with high moisture are easy contaminated by the microbial infection; Dried BCP and BB are better store at lower temperature in the dark at room temperature; the storage condition should be choose store at $-18,-20,4^{\circ} \mathrm{C}$ or stored in sterilized containers or bags and stored at $4^{\circ} \mathrm{C}$ in the dark at room temperature $\left( \pm 20^{\circ} \mathrm{C}\right)$, homogenized the BCP samples and stored them under argon at $-24^{\circ} \mathrm{C}$;

- Raw propolis was better stored at lower temperature.

- The collected honey samples can be stored at 4 or $20^{\circ} \mathrm{C}$;

- The storage temperature for the RJ is controversy for the water content in it; storage temperature of $4^{\circ} \mathrm{C}$ is appropriate for raw RJ, for the lyophilized RJ temperature could be at room temperature, and at $4-8$ or $<-18^{\circ} \mathrm{C}$ for different days.

\section{PROCESSING TECHNIQUES APPLIED IN BEE PRODUCTS}

Processing techniques influence food composition and structure, including the flavor, physical properties, functions, and in turn the product quality (138). The processing techniques applied in the bee products are shown in the Figure 3. Drying techniques is essential for controlling the moisture of BCP and BB after harvest. For RJ, the production and harvest are distinct from that of other bee products as the machines used are different. Special techniques with minimum impact on the health of bees are needed to collect a considerable quantity of BV (139). For the other bee products, extraction, separation, purification, and identification procedures are in common. Quality control techniques are important applied in BCP, BB, RJ, honey, and propolis. The characters and the effects of each drying techniques are shown in Supplementary Table 2 and Figure 4.

\section{Drying Techniques}

Fresh BCP contains 20-30\% water. The higher humidity makes $\mathrm{BCP}$ easily contaminated by microorganisms such as bacteria and yeast. To prevent spoilage and preserve the maximum quality, the BCP must be harvested daily and frozen immediately. The drying of $\mathrm{BB}$ is also an important technological process that affect the quality of the bee product (140). Among the four steps procedure (drying, segmenting, filtering, and disinfecting) (33) for the $\mathrm{BB}$ industrial collection, drying is the most important process. 


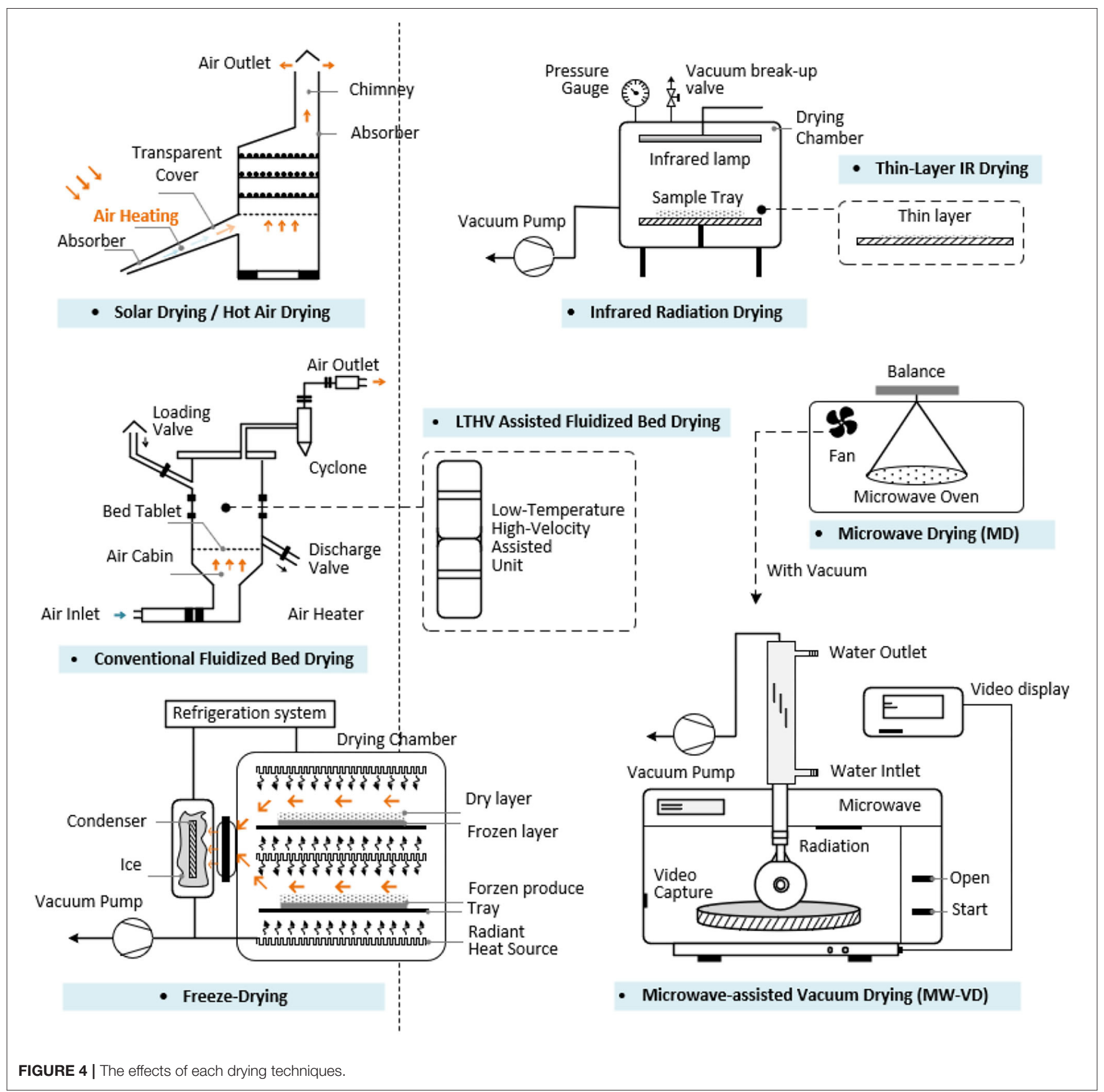

\section{Traditional Drying Techniques}

Traditional drying methods include freezing, solar drying and in a hot air chamber. The bioactive compound content like carotenoid and phenol content in dry BCP and the antioxidant activity ( $0.85 \mathrm{mmol}$ Trolox/g) did not change substantially from the initial raw BCP drying by solar dehydration (141).

Compared with the sun drying, hot air drying has a reasonably shorter processing time, more effective sanitary conditions, lower risk of microbial contamination, and better control of drying conditions. It has been used for the dehydration of food and agricultural products in an industrial scale drying applications
(118). But hot air drying processing has an effect on the BCP quality and organoleptic characteristics, physicochemical properties, morphological structure (118). BCP dried at 40 and $50^{\circ} \mathrm{C}$ showed a marked increase in acidity, flavonoids, phenolics, and antioxidant activity after thermal treatment, and a loss of carotenoids, while the most adequate temperature for BCP drying is at $60^{\circ} \mathrm{C}(142)$.

In comparison with the convective drying, the cyclic convective drying of Perga is not only highly effective to reduce the moisture content and can also reduce energy costs by more than two times. The low speed of the cyclic drying process avoids 
overheating of the Perga and ensures the safety of biologically active substances (143).

FD is considered the most gentle drying method, with fast rehydration rates and high rehydration capacity, as it causes negligible damage to the product microstructure and thus maximum preserve the physical and chemical properties (144). FD influenced the rutin level of the honeybee-collected chestnut pollen, when treated for 420 and $540 \mathrm{~min}$, rutin and amino acid parameters were maintained in the range that required for the standardization of commercial BCP in the European market (145).

Currently, the freezing and FD methods are often used to preserve RJ. The optimal mathematical model was build and suitably used for calculating and setting up the technological mode of the RJ FD processing (146). During the FD process, the nutritional properties of RJ well-preserved, but FD RJ is highly susceptible to Maillard reaction (MR) and cannot meet the product quality and stability requirements (130). The final product of the FD process of RJ had a very good quality (147).

\section{Innovative Drying Techniques}

IR radiation drying is an innovative drying technique which can be applied in BCP samples. IR radiation greatly affected the drying and quality characteristics of the samples, especial the color. Surface morphology changes of BCP grains a lot with the increasing IR intensity.

Drying the $\mathrm{BCP}$ using IR radiation at $50 \mathrm{~W}$ better retained its quality characteristics than treated at other power levels (148). An IR heating-assisted fluidized bed dryer was applied in the BCP drying process to ensure physical and microbiological stability. The drying condition is more smoother than thin-layer IR drying that prevented color degradation, thus conserving product quality of the $\mathrm{BCP}$, and compared with the conventional fluidized bed drying the specific energy consumption was 52\% lower (51).

The developed model of $\mathrm{BB}$ drying with IR heating or the combine use of the IR heating with the convection drying was built using the COMSOL Multiphysics. The geometric dimensions of the drying chamber, the trays with the product, the thermal and physical properties of air and $\mathrm{BB}$ should be considered (140).

Microwave heating can be an efficient drying method. Compared to the conventional drying, microwave can lead to a faster process and obtain better quality of the dried material at the same cost (149). Irradiating fresh BCP with microwaves under vacuum can effectively reduce the water content without thermally deteriorating important bioactive compounds (26). Microwave drying was important for the BCP conservation, but the microwave treatment showed a damaging action on tocopherols (150) and proline content at the highest level treatment (151).

A domestic digital microwave oven $(505 \mathrm{~mm} \times 574 \mathrm{~mm} \times$ $376 \mathrm{~mm}$ in size) (Arcelik MD 599, Turkey) with technical features of $230 \mathrm{~V}$ and $50 \mathrm{~Hz}$ was used for the BCP drying. Operating at a frequency of $2,450 \mathrm{MHz}$ with $180 \mathrm{~W}$ microwave power level retained its quality (152). BCP dried using microwave-assisted vacuum drying (MW-VD) had higher antioxidant activity than hot air drying method (HAD), irrespective of the pressure or power level (153).

Different drying processes (FD, MWD, and HAD) affect the polyphenol, flavonoid, and amino acid contents of the BCP (chestnut, willow, ivy). All drying techniques led to a depletion of flavonoids in willow BCP; Whereas, FD and MWD did not affect the flavonoid content in the ivy pollen during storage, the amino acid-related quality of BCP was efficiently preserved up to 6 months. MWD ensured the highest flavonoid content even after 6 months (154). However, low temperature high velocity (LTHV)assisted fluidized bed drying method preserve the bioactive components of $\mathrm{BCP}$ (155), it can be applied especially drying $\mathrm{BCP}$ as a bioactive food at $4^{\circ} \mathrm{C}$.

\section{Wall-Breaking}

BCP cell wall contains two distinct layers. The outer layer exine is made of sporopollenin, which provides chemical resistance and preserves the bioactive compounds in it. The inner layer is intine, which is composed of cellulose and similar to that of a plant cell wall (156). Wall breaking methods like physical, chemistry, biological and combinations are used to break the $\mathrm{BCP}$ cell wall (157). The effects of each of the BCP cell wall breaking and some related research are shown in the Figure 5 and Table $\mathbf{1 .}$

Several biotechnological solutions have been proposed for $\mathrm{BCP}$ processing to increase the accessibility to nutrients for intestinal absorption, the fermentation being one of the most potential method. Fermented BCP is enriched in bio accessible phenolics with recognized bioactivity in humans (159). The yeast-fermented BCP greatly increased the number of active ingredients such as phenolic compounds, nicotinic acid, oligopeptides, nicotinamide, fatty acids, riboflavin, and free amino acids (158). Fermented BCP is distinguished by its higher nutritional value and better digestibility because the BCP cell walls are partly destroyed during the fermentation process (69).

In addition to the role of microorganisms, the use of proper enzymes is a good way to hydrolyze the wall of the BCP. Enzyme hydrolysis is safe, inexpensive, and easy to apply. Pepsin, trypsin, and papain hydrolysates from $\mathrm{BCP}$ are beneficial not only in health food diets, but for special patients with diseases as cancer, cardiovascular, and diabetes. Thus, honeybee-collected pollen peptides can be used in various fields of medicine and food technology (161). Compared to other BCP modification strategies, proteases improved the $\mathrm{BCP}$ protein content by $\sim 13-$ $18 \%$, phenolics $83-106 \%$, flavonoids $85-96 \%$, and all essential amino acids were increased, even the antioxidant activity increased up to $68 \%(160)$.

Physical processing technology also resulted in the breaking of the BCP cell wall. Ultrasonication and high shear (US-HS) technique was applied to break the cell walls of five species of BCP. The digestibility of protein and crude fat, amino acids, and reducing sugars increased to more than $80 \%$ and nearly 1.5 -and 2 times, respectively (162). Besides, an ultrasonic temperature difference was applied to treat the bee pollen under specified conditions: the ratio of water to material $20 \mathrm{~mL} / \mathrm{g}$, ultrasonic power $400 \mathrm{~W}$, temperature difference $90^{\circ} \mathrm{C}$, ultrasonic treatment time $40 \mathrm{~min}$. The extracts after the wall-breaking are 


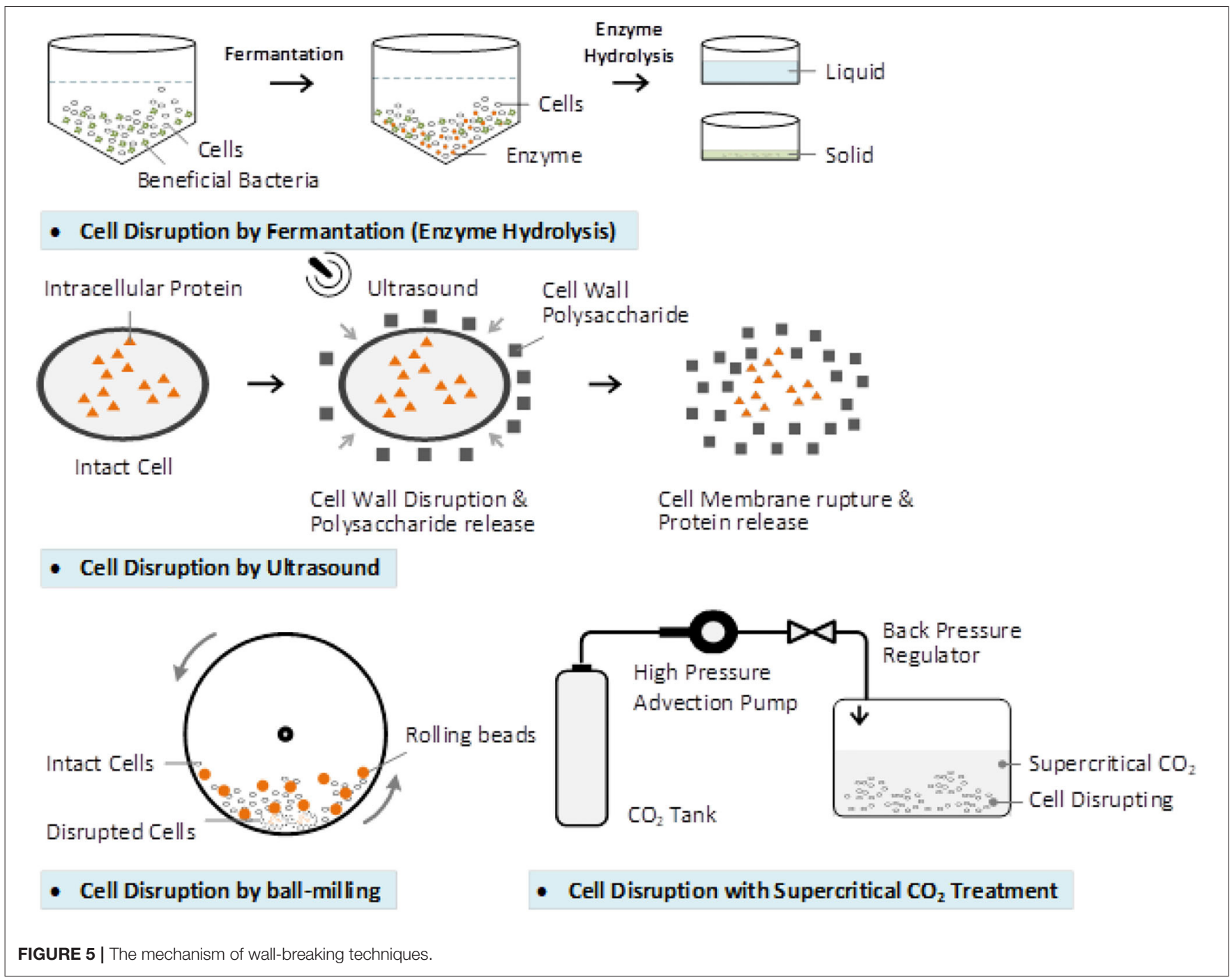

potential to be developed as effective functional ingredients as immunomodulatory (163).

Ultrasonic and ball-milling treatment especially the ultrasonic treatment affected nutritional component release and thus change the antioxidant activities in vitro and in vivo of the rose BCP (29). Supercritical carbon dioxide $\left(\mathrm{CO}_{2}\right)$ could be a feasible and a promising cell wall-breaking method applied to the BCP (156).

\section{Other Processing Techniques Shredding of the Bee Pollen}

The technology of shredding was optimized in the BCP to obtain high-quality powder by its technological and physical-chemical indicators. By using modern types of shredders, it is possible to shred BCP to particles with sizes ranging from 120 to $8 \mu \mathrm{m}$. Technological parameters of shredding BCP in a mill-mortar: speed is $70-80 \mathrm{rpm}\left(\mathrm{min}^{-1}\right)$, treatment duration is $6 \mathrm{~min}$, and the batch weight is $150 \mathrm{~g}$ (164).

\section{Collection and Membrane Ultrafiltration of BV}

To collect a considerable quantity of BV, Markovic and Molnar (1963) used electroshocks and squeezed to induce a bee sting (165). A method modified from Benton et al. (166) was used to collect the liquid fraction of the BV in sufficient quantity to facilitate chemical analysis. Carpena et al. also used electroshock traps to collect more BV (139). BV from free-living honeybees (A. mellifera) was obtained using a collecting device (Chung-Jin Biotech Ltd., Ansan, Korea) or Bee Whisper 0412 BV collector (IGK Electronics, Bulgaria). The collectors were installed at the entrance of the hive to collect crude BV $(167,168)$.

Hermia's models were used to predict the fouling mechanisms during cross-flow ultrafiltration (UF) (169) of BV in a $10 \mathrm{kDa}$ regenerated cellulose membrane $(170,171)$.

\section{Production and Harvesting of RJ}

The conventional method of producing RJ usually involves five steps: cleaning queen cells, grafting larvae, inserting RJ production frames, supplementary grafting, and harvesting. A 
TABLE 1 | BCP wall-breaking.

\begin{tabular}{|c|c|c|c|c|}
\hline Bee products & Wall-breaking method & Conditions and results & Advantages and disadvantages & References \\
\hline Rose BCP (China) & Ball-milling treatment & $\diamond$ Milling for $3 \mathrm{~min}$ at $25 \mathrm{~Hz}$ frequency & & (29) \\
\hline Rose BCP (China) & Ultrasonic treatment & $\begin{array}{l}\diamond \text { At acoustic power of } 600 \mathrm{~W} \text { and } \\
\text { frequency of } 20 \mathrm{kHz} \text { treating } 4 \mathrm{~h} \text { at } 25^{\circ} \mathrm{C}\end{array}$ & $\begin{array}{l}\text { More effective in nutritional } \\
\text { components release } \\
=\text { Causes more losses in vitamin B1 and } \\
\text { vitamin C }\end{array}$ & (29) \\
\hline Rape BCP (China) & Supercritical carbon dioxide & $\begin{array}{l}\diamond \text { More effective with higher } \mathrm{CO}_{2} \text { pressure } \\
\diamond \text { Optimum oil yield was obtained } 5.98 \% \\
\text { of dry } \mathrm{BCP} \\
\diamond \text { Optimum conditions: } 39.2 \mathrm{MPa} \text { and } \\
54.7^{\circ} \mathrm{C} \text { and } \mathrm{CO}_{2} \text { flow rate of } 17.1 \mathrm{~L} / \mathrm{h} \\
\text { after treated with supercritical } \mathrm{CO}_{2} \\
\text { for } 2 \mathrm{~h} \text {. }\end{array}$ & $\begin{array}{l}>\text { Under low temperature with short time } \\
>\text { Higher selectivity } \\
>\text { No solvent residue in the extract }\end{array}$ & $(156)$ \\
\hline $\begin{array}{l}\text { Rape BCP, Brassica } \\
\text { campestris L., BP } \\
\text { (China) }\end{array}$ & $\begin{array}{l}\text { Microbial fermentation } \\
\text { (lactic acid bacteria, yeast, } \\
\text { the two mixed) }\end{array}$ & $\begin{array}{l}\diamond 83.5 \% \text { reduction in fructose and } 87.4 \% \\
\text { glucose reduction after } \\
\text { yeast-fermented } \\
\diamond \text { Oligopeptides, fatty acids and phenolic } \\
\text { compounds increased by } 68.8,18.2, \\
\text { and } 9.3 \%\end{array}$ & & $(158)$ \\
\hline $\begin{array}{l}\text { Bee-collected pollen } \\
\text { (BCP) flowers of ivy } \\
\text { (Italy) }\end{array}$ & $\begin{array}{l}\text { Fermented with selected } \\
\text { microbial }\end{array}$ & $\begin{array}{l}\diamond \text { Incubated kunkeei strains and } \mathrm{H} \text {. } \\
\text { uvarum AN8Y27B at } 30^{\circ} \mathrm{C} \text { for } 216 \mathrm{~h}\end{array}$ & $\begin{array}{l}\text { Markedly increased the number of } \\
\text { volatile compounds (VOC) }\end{array}$ & $(159)$ \\
\hline BCP (Colombia) & Enzymatic hydrolysis & $\begin{array}{l}\diamond \text { Proteases improved the protein content } \\
\text { by } \sim 13-18 \%, \text { phenolics } 83-106 \%, \\
\text { flavonoids } 85-96 \%, \\
\diamond \text { increased all essential amino acids and } \\
\text { antioxidant activity up to } 68 \%\end{array}$ & & $(160)$ \\
\hline $\begin{array}{l}\text { Pollen Cistus } \\
\text { ladanifer (Japan) }\end{array}$ & Enzymatic hydrolysates & $\begin{array}{l}\diamond \text { Three types of enzymes (pepsin, } \\
\text { trypsin, and papain) } \\
\diamond \text { high content of proteins and phenolic } \\
\text { compounds after } \\
\text { enzymatic hydrolysates }\end{array}$ & & $(161)$ \\
\hline
\end{tabular}

BCP, Bee Collected Pollen; VOC, volatile compounds; $\mathrm{CO}_{2}$, carbon dioxide.

new method based on a device that obviates grafting and is therefore convenient and efficient $(172,173)$.

Currently, electroshock technology is widely used for the BV collection. For the RJ production technology, the new method is convenient and efficient.

\section{Extraction, Separation, and Purification Techniques}

Considering the current demand for healthy and natural food, some hive products have been attracting the commercial interest recently, for several health-promoting compounds contained, making them one of the most widely consumed food supplements. Extraction and separation techniques are essential for obtaining large quantities of high-quality active ingredients. Recent study (5 years) about the sugars/polysaccharide extraction, separation and purification techniques were shown in the Table 2 .

\section{Sugars/Polysaccharide}

Carbohydrate was the largest fraction that contained in the honey and BCP. The concentration and carbohydrates ratio may play a role in the identification of geographical origins and the monofloral of the honeys (180). The main compound in honey is carbohydrates, which contribute about $95-97 \%$ of its dry weight $(41,48)$. Predominantly, monosaccharides such as glucose and fructose $(40,49,50,181)$, mannose, and rhamnose are common in honey. In addition, disaccharides, trisaccharide, and tetra saccharides have also been identified in honey $(133,179)$. The beneficial effects of honey on human health have long been recognized (180). BCP contains about $15-60 \%$ carbohydrates, which represent the most fraction in the BCP (182). Monosaccharides are the major source of metabolism energy, polysaccharides store energy, and are also the structural components (183). Glucose and fructose are the most abundant reducing sugars in BCP (9). In addition, other carbohydrates such as disaccharides (sucrose), monosaccharides (pectin, glucan, and arabinogalactan), oligosaccharides, and dietary fiber were also found in $\mathrm{BCP}(176,178)$. The main sugars in RJ are fructose and glucose in relatively constant proportions similar to those of honey (13).

The extraction methods of polysaccharides focus on the traditional method, after $96 \%$ ethanol (175) or ultrasonic assist (30) solvent to skim the lipid, hot water $(163,176-178)$ is 


\begin{tabular}{|c|c|c|c|c|c|}
\hline $\begin{array}{l}\text { Bee products } \\
\text { reference }\end{array}$ & Sugars or polysaccharides & Extraction method & Determine method & Separation/purification & Identification/analysis \\
\hline $\begin{array}{l}\text { Honey (France) } \\
\text { (174) }\end{array}$ & - & & & $\begin{array}{l}\text { SPE filtered on } 0.45 \mu \mathrm{m} \\
\text { syringe filter }\end{array}$ & HPAEC-PAD Analysis \\
\hline $\begin{array}{l}\text { BCP (China) } \\
(20)\end{array}$ & - & & $\begin{array}{l}\text { Phenol-sulfuric acid } \\
\text { method }\end{array}$ & DEAE-52 cellulose column & $\begin{array}{l}\text { Fourier } \\
\text { transformed-infrared } \\
\text { (FT-IR) }\end{array}$ \\
\hline $\begin{array}{l}\text { BCP (China) } \\
(175)\end{array}$ & - & $\begin{array}{l}90 \% \text { ethanol solution; } \\
\text { Next soaked in distilled } \\
\text { water }\end{array}$ & Phenol-sulfuric acid & & Gas chromatography \\
\hline $\begin{array}{l}\text { BCP (China) } \\
\text { (163) }\end{array}$ & - & $\begin{array}{l}\text { Temperature-difference } \\
\text { ultrasonic treatment; } \\
\text { extraction was carried out } \\
\text { at } 85^{\circ} \mathrm{C}\end{array}$ & & $\begin{array}{l}\text { Quadruple volume of } \\
\text { absolute ethanol } \\
\text { precipitates }\end{array}$ & \\
\hline $\begin{array}{l}\text { BCP (China) } \\
\text { (176) }\end{array}$ & $\begin{array}{l}\text { Arabinose, xylose, galactose, mannose, ribose, } \\
\text { rhamnose, galacturonic acid, glucose, and in a molar } \\
\text { ratio of 1.03: } 0.08: 0.67: 0.38: 0.09: 0.17: 0.64: 0.22\end{array}$ & $\begin{array}{l}\text { With distilled water } \\
\text { extracted three times at } \\
85^{\circ} \mathrm{C} \text { for } 2 \mathrm{~h} \text { each }\end{array}$ & Phenol-sulfuric acid & $\begin{array}{l}\text { A quadruple volume of } \\
\text { absolute ethanol } \\
\text { precipitates }\end{array}$ & $\begin{array}{l}\text { High-performance gel } \\
\text { permeation } \\
\text { chromatography }\end{array}$ \\
\hline $\begin{array}{l}\text { BCP of } \\
\text { Nelumbo } \\
\text { nucifera } \\
\text { (China) (177) }\end{array}$ & $\begin{array}{l}\text { Rha (11.5\%), Ara (29.7\%), GalA (12.0\%), Gal (41.2\%), } \\
\text { 1.5-L-Ara (25.6\%), 1,6-D-Gal (18.3\%), t-D-Gal (12.0\%) }\end{array}$ & Hot water extraction & & $\begin{array}{l}\text { DEAE-cellulose, } \\
\text { Sepharose CL-6B }\end{array}$ & $\begin{array}{l}\text { GC-MS } \\
13 C-N M R\end{array}$ \\
\hline $\begin{array}{l}\text { BCP (China) } \\
(178)\end{array}$ & $\begin{array}{l}\text { Two sub-fractions: RBPP-N and PBPP-P (yield 23.4\% } \\
\text { and } 35.8 \% \text { ) and PBPP-P (yield 35.8\%); Glc (glucose } \\
35.0 \% \text { ) is special contained in RBPP-N, which might be } \\
\text { glucan and arabinogalactan (AG); } \\
\text { Ara (arabinose: } 37.8 \%, 51.8 \% \text { ), and Gal (galactose: } \\
27.2 \% \text { and } 21.0 \% \text { ) are identify out in both subfractions, } \\
\text { Rha (rhamnose: } 5.4 \% \text { ), and Gal A (galacturonic acid: } \\
\text { 18.9\%) were special contained in RBPP-P, might be } \\
\text { monosaccharides for pectin; }\end{array}$ & $\begin{array}{l}\text { Hot water extraction and } \\
\text { ethanol precipitation }\end{array}$ & & DEAE-cellulose & $\begin{array}{l}\text { 13C-NMR, FT-IR } \\
\text { spectroscopy }\end{array}$ \\
\hline $\begin{array}{l}\text { RJ (Greece) } \\
\text { (132) }\end{array}$ & Glucose, fructose, and sucrose & & & $\begin{array}{l}\text { Zorbax Carbohydrate } \\
\text { Analysis Column }\end{array}$ & HPLC-RID technique \\
\hline $\begin{array}{l}\text { Honey } \\
\text { (European) } \\
\text { (China) (179) }\end{array}$ & $\begin{array}{l}\text { Monosaccharides (glucose, fructose, rhamnose, } \\
\text { mannose); } \\
\text { Disaccharides (sucrose, -trehalose, turanose, maltose, } \\
\text { isomaltose, gentiobiose nigerose, maltulose, palatinose, } \\
\text { melibiose, melezitose, and kojibiose); } \\
\text { Trisaccharides (melezitose, maltotriose, panose, } \\
\text { raffinose, isomaltotriose, erlose, and 1-kestose). }\end{array}$ & & & & NMR \\
\hline $\begin{array}{l}\text { Honey (Italy) } \\
\text { (133) }\end{array}$ & $\begin{array}{l}\text { Seven monosaccharides; } \\
\text { Four trisaccharides; eight Disaccharides; } \\
\text { One tetrasaccharide; }\end{array}$ & & & $\begin{array}{l}\text { CarboPac PA10 }{ }^{\mathrm{TM}} \\
\text { column (Thermo Scientific, } \\
2 \times 250 \mathrm{~mm}, 10 \mu \mathrm{m}) \\
\text { equipped with a } \\
\text { CarboPac PA10 } 10^{\mathrm{TM}} \text { guard } \\
\text { column }(2 \times 50 \mathrm{~mm})\end{array}$ & $\begin{array}{l}\text { High-performance } \\
\text { anion-exchange } \\
\text { chromatography coupled } \\
\text { to a mass spectrometry } \\
\text { detector }\end{array}$ \\
\hline
\end{tabular}

$35.0 \%$ is special contained in RBPP-N, which might be

Next soaked in distilled

Temperature-difference

asonic treatment;

xtraction was carried out

With distilled water

$85^{\circ} \mathrm{C}$ for $2 \mathrm{~h}$ each

Rha (11.5\%), Ara (29.7\%), GalA (12.0\%), Gal (41.2\%),

BCP (China) 


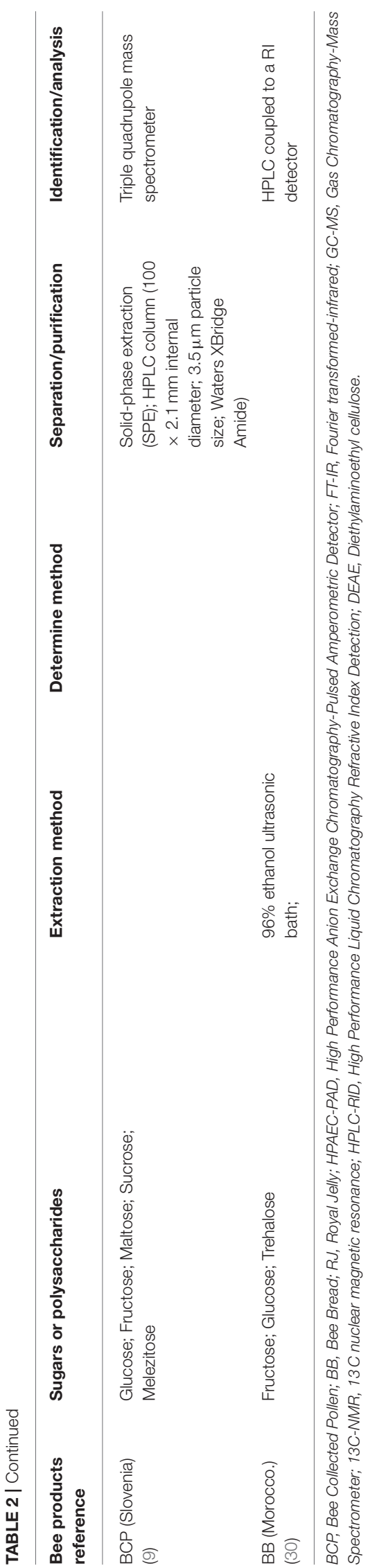

used to extract the polysaccharide, and then a quadruple volume of absolute ethanol precipitates $(163,176,178)$. Solidphase extraction $(9,174)$ as well as DEAE-52 cellulose column (20, 177, 178), Zorbax Carbohydrate Analysis Column (132) and other columns were used to separate the polysaccharides. High-performance liquid chromatography (HPLC), HPAECPAD (174), Fourier transform infrared (FT-IR) spectroscopy $(20,178)$, gas chromatography (GC) (175), high-performance gel permeation chromatography (176), GC-MS (177), ${ }^{13} \mathrm{C}$ NMR (177-179), and HPLC-RID (132) techniques were used to identify the polysaccharides.

\section{Lipid or Fatty Acids}

Besides the carbohydrates and proteins in the BCP, lipids are the third-largest constituent, lipids are vital for the generation of RJ (3). Among bee products, lipids from BCP, BB, RJ, and Beeswax have been widely studied in recent 5 years. The fatty acids identified are listed in Table 3. Lipids from bee products extracted using a Soxhlet extractor $(62,186)$ or extracted with a simple solvent. The extraction solvents such as petroleum ether (124), hexane:isopropanol (184), and diethyl ether:petroleum ether $(2: 1, \mathrm{v} / \mathrm{v})(186,187)$ diethyl ether/isopropanol, chloroform:methanol (1:1), chloroform:isopropanol (4:1) (150), methanol/diethyl ether/isopropanol 50/1 (v/v) (188), heptane (112), $\left(\mathrm{CH}_{2} \mathrm{Cl}_{2}: \mathrm{MeOH}\right)(2 / 1 \mathrm{v} / \mathrm{v})$ extraction (189), and $\mathrm{n}$-hexane $(111,127,190)$ are used these years. Gravimetric method was used to determine the total lipid content in all cases.

Traditional extraction techniques with the disadvantages as the long extraction time, loss of the volatile compounds, toxic solvent residues, and the unsaturated compounds degraded due to heat (191). In certain cases, it can be resolved by using supercritical fluid (carbon dioxide) extraction (SFE).

For the identification technologies, thin-layer (184), capillary gas chromatography, gas chromatography $(111,114,190)$, GCFID $(16,30,186,190)$, GC/MS $(36,127,187,190,192), \mathrm{GC}$, and 5,973 MSD mass spectrometry (113), are the preferred techniques for determining fatty acids. The samples detected using GC-MS should be firstly converted into their corresponding methyl esters (FAMEs) using a transesterification procedure, chromatographic data obtained are more robust and reproducible (193).

Total lipid content of the BCP varying from 1 to $13 \%$ from different botanical species. The lipid fraction of BCP contains mainly carotenoids, sterols, and fatty acids (194). Fatty acids are important nutritional substances for living organisms, especially those of the $\omega-3$ series. The fatty acid of the BCP with the functional role as an anti-atherogenic food for human metabolism (150). Nearly 20 fatty acids have been reported in BCP from $\mathrm{C} 4$ to $\mathrm{C} 24$, among which $\omega-3$ fatty acids are dominant $(36,62,111-114,150,184)$. In beeswax, fatty acids were identified from C12:0 to C36 (189). Approximately 80$90 \%$ of the RJ fatty fraction is composed of free fatty acids. The major fatty acid from the RJ were trans-10-hydroxy-2decenoic acid [10-hydroxydecanoic acid (HDA)], and other four suspect fatty acids decanedioic acid, 2-dodecenedioic acid, decanoic acid, and decanedioic acid were also detected using the liquid chromatography-high resolution mass spectrometry (HRMS) method (188). 
TABLE 3 | Lipid extraction, separation purification, and identification of the fatty acids from the bee products (2017-2021).

\begin{tabular}{|c|c|c|c|c|}
\hline $\begin{array}{l}\text { Bee products } \\
\text { reference }\end{array}$ & Compounds & Extraction method & Separation/purification & Identification/analysis \\
\hline $\begin{array}{l}\text { BCP (Italy) } \\
(184)\end{array}$ & $\begin{array}{l}\text { Ten saturated and the other unsaturated } \\
\text { C8:0; C13:0; C15:0; C16:0; C17:0; C18:0; C18:1; C18:2n-6t: C18: 2n-6C; } \\
\text { C18: 3n-6; } \\
\text { C18: 3n-3; C20:0; C20:1; C20:2; C22:0; C22:1; C23:0; C24:0 N-6PUFA; } \\
\text { n-3 PUFA }\end{array}$ & Hexane:isopropanol & Silica Gel 60, 230/400 mesh & $\begin{array}{l}\text { Thin-layer chromatography } \\
\text { Gas chromatography }\end{array}$ \\
\hline $\begin{array}{l}\text { BCP (China) } \\
\text { (185) }\end{array}$ & Phospholipids and polyunsaturated fatty acids & Folch method & $\begin{array}{l}\text { XSelect CSH C18 } 100 \\
\times 2.1 \mathrm{~mm}, 2.5 \mu \mathrm{m} \text { column } \\
\text { (Waters) and CORTECS } \\
\text { C18 } 100 \times 2.1 \mathrm{~mm}, 2.7 \mu \mathrm{m} \\
\text { column (Waters) }\end{array}$ & $\begin{array}{l}\text { UPLC-Q-Exactive Orbitrap/ } \\
\text { MS }\end{array}$ \\
\hline $\begin{array}{l}\text { BCP (Portugal) } \\
\text { (124) }\end{array}$ & - & $\begin{array}{l}\text { Petroleum ether using an } \\
\text { automatic Soxtec device }\end{array}$ & Gas chromatography & Flame ionization \\
\hline $\begin{array}{l}\text { BCP (Italy), } \\
\text { (Spain), } \\
\text { (Colombia) } \\
\text { (186) }\end{array}$ & - & $\begin{array}{l}\text { Soxhlet extraction using a } \\
\text { Soxtec HT } 1043 \text { system } \\
\text { ether:petroleum ether }(2: 1 \text {, } \\
\text { v/v) }\end{array}$ & $\begin{array}{l}\text { Omegawax } 320 \text { capillary } \\
\text { column; }\end{array}$ & GC-FID \\
\hline RJ (Italy) (187) & - & Diethyl ether/iso-propanol & $\begin{array}{l}\text { Fused silica capillary column } \\
\text { SE-52 }\end{array}$ & $\begin{array}{l}\text { Capillary Gas } \\
\text { Chromatography } \\
\text { GC/MS }\end{array}$ \\
\hline $\begin{array}{l}\text { BCP (Brazil) } \\
\text { (57) }\end{array}$ & & Soxhlet extraction & & \\
\hline $\begin{array}{l}\text { BCP (Italy) } \\
(150)\end{array}$ & (150) $\omega-6$ fatty acids $\omega-3$ fatty acids and carotenoids & $\begin{array}{l}\text { Chloroform:methanol (1:1) } \\
\text { solution solid-phase } \\
\text { extraction } \\
\text { Chloroform: } \\
\text { isopropanol (4:1) }\end{array}$ & & $\begin{array}{l}\text { GC2010 Shimadzu gas } \\
\text { chromatograph } \\
\text { High polar fused-silica } \\
\text { capillary column }\end{array}$ \\
\hline $\begin{array}{l}\text { RJ (Greece) } \\
(188)\end{array}$ & $\begin{array}{l}\text { 10-DHA; 2-Dodecenedioic acid; Decanoic acid; Decanedioic acid; } \\
\text { 3-Hydroxydecanoic acid; Decanedioic acid; }\end{array}$ & $\begin{array}{l}\text { Methanol/ Diethyl } \\
\text { ether/isopropanol 50/1 (v/v) }\end{array}$ & & $\begin{array}{l}\text { Liquid chromatography-high } \\
\text { resolution MS }\end{array}$ \\
\hline $\begin{array}{l}\text { BCP/BB } \\
\text { (Turkey) (113) }\end{array}$ & $\begin{array}{l}\text { Caproic acid; Caprylic acid; Myristic acid; Palmitoleic acid; Stearic acid; } \\
\text { Oleic acid; Linoleic acid; Linolenic (ALA) acid; Arachidic acid; Eicosenoic } \\
\text { acid; Eicosadienoic acid; Behenic acid; Erucic acid; Tricosanoic acid; } \\
\text { Lignoceric acid; Palmitic acid; Nervonic acid; Docosahexaenoic acid; Capric } \\
\text { acid; Lauric acid; Heneicosanoic acid; Heptadecanoic; }\end{array}$ & $\begin{array}{l}\text { Ethanol (95\%), } \\
\text { ultrasonic-assisted } \\
\text { extraction; AOAC method } \\
\text { Soxhlet extraction }\end{array}$ & $\begin{array}{l}\text { A DB- } 2360 \mathrm{~m} \times 0.25 \mathrm{~mm} \\
\text { ID, } 0.15 \mu \mathrm{m}(\mathrm{J} \& \mathrm{~W} \\
122-2,361) \text { column }\end{array}$ & $\begin{array}{l}\text { GC and } 5,973 \text { MSD mass } \\
\text { spectrometry }\end{array}$ \\
\hline $\begin{array}{l}\text { BCP (Serbia) } \\
(112)\end{array}$ & $\begin{array}{l}\text { C18:0; C12:0; C14:0; C15:0; C16:0; C16:1; C17:0; C18:0; C18:1n-9; C18: } \\
\text { 2n-6; C18: 3n-6; C20:0; C21:1; C20:2; C20:3n-6; C20:3n-3; C22:0; } \\
\text { C22:5n-3; C24:0; }\end{array}$ & $\begin{array}{l}\text { Ultrasonic assist heptane } \\
\text { extraction for } 15 \mathrm{~min}\end{array}$ & $\begin{array}{l}\text { Filtered through filter paper } \\
\text { (Whatman No. 2); } \\
\text { cyanopropyl HP-88 column }\end{array}$ & $\begin{array}{l}\text { GC capillary analysis with } \\
\text { FID }\end{array}$ \\
\hline $\begin{array}{l}\text { BCP (Korea) } \\
(62)\end{array}$ & $\begin{array}{l}\text { C6:0; C8:0; C10:0; C12:0; C13:0; C14:0; C15:0; C16:0; C16:1; C17:0; } \\
\text { C18:0; C18:1; C18:2 n-6; C18:3 n-6; C18:3 n-3; C20:0; C20:1; C20:2; } \\
\text { C20:3 n-3; C20-4 n-6; C21:0; C22:0; C22:1; C22:2; C22:6 n-3; C23:0; } \\
\text { C24:0; C24:1; }\end{array}$ & Soxhlet extraction & & GC-FID \\
\hline $\begin{array}{l}\text { BCP (Turkey) } \\
\text { (114) }\end{array}$ & $\begin{array}{l}\text { Lauric; Myristic; Palmitic; Linolelaidic; Arachidic; Linolenic; Behenic; } \\
\text { Arachidonic Stearic; Elaidic; Oleic; Linoleic; }\end{array}$ & & FID and capillary column & GC \\
\hline
\end{tabular}


reference amino]-, trimethylsilyl ester; Linoleic acid ethyl ester; ethyl ester;9,12-Octadecadienoic acid (Z, Z)-, trimethyl silyl ester; alpha.-linolenic acid, trimethyl silyl ester; Dodecanoic acid, tetradecyl ester; 9,12,15-Octadecatrienoic acid;

BCP (India)

BB (Romania)

(127)
C4:0 Butyric acid: C6:0 Caproic acid; C8:0 Caprylic acid; C10:0 Capric acid; C11:0 C12:0 Lauric acid; C14:0 Myristic acid; C15:0 Pentadecanoic acid; C16:0 Palmitic acid; C16:1, cis- $\Delta 7$ ( $\omega-7)$ Palmitoleic acid; C17:0 Heptadecanoic acid; C17:1, cis- $\Delta 7$ ( $\omega-7)$ Heptadecenoic acid; C18:0 Stearic acid; C18:1, trans- $\Delta 9(\omega-9)$ Elaidic acid; C18:1, cis- $\Delta 9(\omega-9)$ Oleic acid; C18:1, cis- $\Delta 6$ ( $\omega-12)$ Petroselinic acid; C18:2, cis, cis- $\Delta 9,12(\omega-6)$ Linoleic acid; $C 18: 2$, trans, trans- $\Delta 9,12(\omega-6)$ Linoelaidic acid; $C 18: 3$, cis, cis, cis- $\Delta 9,12,15$ ( $\omega$-3) $\alpha$-Linolenic acid; C18:3, cis, cis, cis- $\Delta 6,9,12(\omega-6)$ $\gamma$-Linolenic acid; C20:0 Arachidic acid; C20:1, cis- $\Delta 11$ ( $\omega$-9) Eicosenoic acid; C21:1, cis- $\Delta 9$ ( $\omega-9)$ Heneicosanoic acid; C20:2, cis, cis- $\Delta 11,14(\omega-6)$ Eicosadienoic acid; C20:3, cis, cis, cis- $\Delta 8,11,14$ ( $\omega$-6) Dihomo- $\gamma$-linolenic cis, cis, cis- $\Delta 5,8,11,14(\omega-6)$ Arachidonic acid; C22:0 Behenic acid; C22:1, cis- $\Delta 13(\omega-9)$ Erucic acid; C22:6, cis, cis, cis, cis, cis, cis- $\Delta 4,7,10,13,16$, 19 ( $\omega$-3) Docosahexaenoic acid; Undecanoic acid; C23:0 Tricosanoic acid; C20:5, cis, cis, cis, cis, cis- $\Delta 5,8,11,14,17$ ( $\omega$-3) Eicosapentaenoic acid;

C24:0 Lignoceric acid; C24:1, cis- $\Delta 15$ ( $\omega-9)$; Nervonic acid;

C4:0 Butanoic acid; C6:0 Hexanoic acid; C8:0 Octanoic acid; C10:0 Decanoic acid; C11:0 Undecanoic acid; C12:0 Dodecanoic acid; C13:0 Tridecanoic acid; C14:0 Tetradecanoic acid; C14:1 (cis-9)

(Z)-tetradec-9-enoic acid; C15:0 Pentadecanoic acid; C15:1 (cis-10)

(Z)-pentadec-10-enoic; C16:0 Hexadecanoic acid; C16:1

(9Z)-hexadec-9-enoic acid; C17:0 Heptadecanoic acid; C17:1

cis-10-heptadecenoic; C18:0 Octadecanoic acid; C18:1

(E)-octadec-9-enoic; C18:1 (Z)-octadec-9-enoic; C18:2 (all-trans-9,12)

Octadeca-9,12-dienoic acid; C18:2 (all-cis-9,12)

(9Z,12Z)-octadeca-9,12-dienoic acid; C18:3 (all-cis-9,12,15)

Octadeca-6,9,15-trienoic acid C20:0 Icosanoic acid; C18:3 (all-cis-6,9,12)

Octadeca-6,9,12-trienoic acid;C20:1 (cis-11) (Z)-icos-11-enoic acid;; C20:2

(all-cis-11,14,) Icosa-11,14-dienoic acid; C20:3 (all-cis-8,11,14)

(8Z,11Z,14Z)-icosa-8,11,14-trienoic acid; C20:3 (all-cis-11,14,17);

(11Z,14Z,17Z)-icosa-11,14,17-trienoic acid; C20:5 (all-cis-5,8,11,14,17);

(5Z,8Z,11Z,14Z,17Z)-icosa-5,8,11,14,17-pentaenoic; C20:4

(all-cis-5,8,11,14); (5Z,8Z,11Z,14Z)-icosa-5,8,11,14-tetraenoic acid; C21:0

Heneicosanoic acid; C22:0 Docosanoic acid; C22:1 (cis-13)

(Z)-docos-13-enoic; C22:6 (all-cis-4,7,10,13,16,19);

Docosa-4,7,10,13,16,19-hexaenoic; C22:2

(all-cis-13,16) Docosa-13,16-dienoic; C23:0 Tricosanoic acid; C24:0

Tetracosanoic acid; C24:1 (cis-15) (Z)-tetracos-15-enoic; acid; C20:3, cis, cis, cis- $\Delta 11,14,17$ ( $\omega$-3) Eicosatrienoic acid; C20:4, cis,
Ethanol extraction solicited

in an ultrasound bath

Agilent mass selective

detector (MSD) 5975C,

HP-5 MS fused-silica

capillary column $(30 \mathrm{~m} \times$

$0.25 \mathrm{~mm}$ I.D, $0.25 \mu \mathrm{m}$ film

thickness)

$\mathrm{n}$-hexane extraction in a

SLB-IL111 capillary column

$(100 \mathrm{~m} \times 0.25 \mathrm{~mm}$ internal

diameter, $0.20 \mu \mathrm{m}$ film

thickness; Sigma-Aldrich

Soxhlet apparatus for $8 \mathrm{~h}$
(GC-MS) 


\section{Proteins, Peptides, and Amino Acids}

Bee products such as $\mathrm{BCP}, \mathrm{BB}$, and $\mathrm{RJ}$ are good sources of protein, which can be consumed as a food supplement to the diet. According to the characteristics of the protein, the extraction solvent usually focuses on distilled water (195), acid extraction (196, 197), phosphate buffer with different $\mathrm{pH}$ ( $\mathrm{pH}$ 7.6) (198), (pH 7.2) (199), or $50 \mathrm{mM}$ Tris-HCl, $\mathrm{pH} 8$ (200). The method for the protein extraction and determination are shown in Table 4.

In most cases, total protein content was the result of nitrogen measurement using the Bradford (199, 200, 208), Kjeldahl, or Micro-Kjeldahl methods $(202,203)$, using $5.60(205,206)$ and 6.25 factor (207). In addition, the Micro BCA Protein Assay Kit $(195,204)$ and Bio-Rad Protein Assay kit $(19,201)$ were used for protein determination.

The crude proteins were analyzed by sodium dodecyl sulfate polyacrylamide gel electrophoresis (SDS/PAGE) (80, 192, 196, 209-213), and western blotting (19, 201), nativePAGE (209), blue native-PAGE or 2-D blue native/SDS-PAGE (204). The tryptic peptides were subsequently determined using two-dimensional polyacrylamide gel electrophoresis (2DPAGE), LC-Chip/ESI-QTOF-MS (80, 199), MALDI-TOF/MS $(214,215)$.

In Tables 4-6, the protein and peptide identified in recent years have been listed. RJ can be consumed by humans as a food integrator, and it has high commercial value because of its nutritional and nutraceutical properties. It is estimated that $50 \%$ of dry RJ constitutes of proteins (237). Majority of RJ proteins (MRJPs) belong to a wider family, nine members have been identified out with molecular weight ranging from 49-87 kDa. The proteins MRJP1, MRJP2, MRJP3, MRJP4, and MRJP5 represent $\sim 82 \%$ of the total proteins present in RJ $(192,238,239)$. MRJP1 has a molecular weight of $57 \mathrm{kDa}$ as a monomer or $350 \mathrm{kDa}$ as a hexamer. MRJP3 is a glycoprotein $(60-70 \mathrm{kDa})$ (195) that has been isolated from RJ. MRJP18 have been characterized by cloning and sequencing their respective cDNAs (240). Royalisin is a $5.5-\mathrm{kDa}$ antibacterial peptide isolated from the RJ and the antimicrobial activity against fungi, gram-negative and gram-positive bacteria have been revealed (234).

Proteins are the second largest amount of nutritional component in BCP and fulfill the nutritional requirements of a honeybee. The peptides from bee products also possess various bioactivities. A novel angiotensin-I converting enzyme (ACE) inhibitory peptide was separated using UF and purified using preparative HPLC, which was derived from rape BCP protein hydrolysate (197). Seven peptide sequences were identified out by the quadrupole time-of-flight mass spectrometry, the three peptide displayed potent anti-inflammatory activity (199). The protein and amino acid concentrations in BCP were variable depending on their botanical origin. Five dominant amino acids (aspartic acid, glycine, glutamic acid, leucine, and alanine) were found in the tested BCP. They constituted $\sim 58.56,56.67,58.80,58.98$, and $58.79 \%$ of the total quantified amino acids from alfalfa, date palm, summer squash, and rape, respectively (205). A novel protein with 42,388 Da from oilseed rape (Brassica napus L.), contained 17 amino acids (215). 
TABLE 4 | The techniques for the protein extraction and determination.

\begin{tabular}{|c|c|c|c|}
\hline Bee products & Extraction method & Determine method & References \\
\hline RJ & & Bio-Rad Protein Assay kit & $(19,201)$ \\
\hline BCP (eastern Saudi Arabia) & & Micro-Kjeldahl method & $(202)$ \\
\hline $\begin{array}{l}\text { Black Dwarf Honey and } \\
\text { BCP (Thailand) }\end{array}$ & & Kjeldahl's method & (203) \\
\hline RJ (Japan) & Dialyzed against distilled water & Micro BCA Protein Assay Kit (Pierce) & $(204)$ \\
\hline RJ (Japan) & $\begin{array}{l}\text { Dissolved in deionized water and } \\
\text { ultracentrifuged }\end{array}$ & Micro BCA Protein Assay Kit & $(195)$ \\
\hline RJ (Pisa) & 50 mM Tris- $\mathrm{HCl}, \mathrm{pH} 8$ & Bradford & $(200)$ \\
\hline RJ (Japan) & Acid extraction & & $(196)$ \\
\hline $\begin{array}{l}\text { BCP (Al-Ahsa Eastern Saudi } \\
\text { Arabia) (Australia) }\end{array}$ & & $\begin{array}{l}\text { Micro-Kjeldahl method using the } 5.60 \\
\text { factor }\end{array}$ & $(205,206)$ \\
\hline BCP (China) & Extracted in phosphate buffer pH 7.6 & & $(198)$ \\
\hline $\begin{array}{l}\text { BCP (Apis mellifera) } \\
\text { (Thailand) }\end{array}$ & $\begin{array}{l}\text { Pretreated with } 1,000 \mathrm{~mL} \text { of } 20 \mathrm{mM} \\
\text { phosphate buffer solution (pH 7.2) } \\
\text { Hydrolyzed by Alcalase, Flavourzyme, or } \\
\text { Neutrase (separately) }\end{array}$ & Bradford & $(199)$ \\
\hline BCP (Bulgaria) & $\begin{array}{l}\text { Extracted with distilled water the } \\
\text { homogenized adjusted } \mathrm{pH} 7.0 \text { hydrolysis }\end{array}$ & Kjeldahl using the equation: $\mathrm{N} \times 6.25$ & $(207)$ \\
\hline $\mathrm{BCP}$ & & Bradford & $(208)$ \\
\hline BCP (China) & $0.1 \mathrm{~mol} / \mathrm{L} \mathrm{HCl}$ solution & & $(197)$ \\
\hline
\end{tabular}

RJ, Royal Jelly; BCP, Bee Collected Pollen; BB, Bee Bread.

$\mathrm{BV}$ is a liquid mixture that contains $88 \%$ water and only $0.1 \mathrm{~g}$ dry weight of a complex mixture of enzymes, peptides, and non-peptide components in one drop (241). Among them, peptides are the main components in BV (242). Approximately $48-50 \%$ of dry venom were the small proteins and peptides. Melittin and PLA2 (229) are the two most abundant compounds in BV. Melittin (a 26-amino acid peptide) is the main biologically active component in $\mathrm{BV}$, which plays an important role in inducing reactions associated with bee stings (100) and it. Melittin and isoforms were also identified using the LC-MS ESI-QTOF system (225-227, 243-246), SDS-PAGE (213), and $\operatorname{HPLC}(100,212)$.

\section{Phenolic and Flavonoid Compounds}

Honey, propolis, $\mathrm{BCP}, \mathrm{BB}, \mathrm{RJ}$, beeswax, and $\mathrm{BV}$ are natural products that have been used for medicine since the ancient times (247).

Phenolic character substances that express the ability to scavenge free radicals, are primarily responsible for the antioxidant capacity of bee products (248). They comprise two main groups of compounds: flavonoids and phenolic acids (249).

The extraction procedure for the phenolics and flavonoids was primarily via solvent extraction using alcohol (methanol or ethanol) and water mixture (76, 250-253); cyclohexane, dichloromethane, butanol, and water (254); petroleum ether, n-butanol, ethyl acetate, and water (255); and acidified water (256). As for the phenolic and flavonoid contents in bee products, the Folin-Ciocalteu method $(57,254,257-259)$ has been predominantly employed. The TFC of the BCP extracts was established (UV-Vis) in most cases using the aluminum trichloride colorimetric assay at $510 \mathrm{~nm}(253,260)$ or aluminum nitrate at $415 \mathrm{~nm}(57,258)$.

The characterization of phenolic compounds has been performed using different methods, especially HPLC (261), and the presence of these compounds in stingless bee honey has been evaluated by matching the retention time of the standard. HPLC with different detectors was also used to identify the phenolic compounds: HPLC with diode array detection (262), and semi-preparative HPLC system with a diode array detector (DAD) (263). High-performance liquid chromatography-mass spectrometry (HPLC-MASS) (264), liquid chromatography with diode array detection coupled with electrospray ionization (ESI) tandem mass spectrometry (LC-DAD-ESI) (265), ultra-high-performance liquid chromatography-diode array detection- mass spectrometry/mass spectrometry (UHPLCDAD-MS/MS) (266), and HPLC-MASS methods have been developed for the characterization of secondary metabolites present in the methanol extract of BCP (267). UHPLC-DADESI-MS, UHPLC-ESI-QTOF, HPLC-PDA, SPME-GC/MS, UHPLC-ESI-QTOF, and related techniques have been widely used to detect many active ingredients (268). The chemical composition of raw propolis was determined using GC-MS (269). Recently, high-speed counter-current chromatography has been used to separate tyrosinase inhibitors from camellia pollen (270, 271). Furthermore, using highspeed countercurrent chromatography and preparative HPLC, nuclear magnetic resonance and mass spectrometry are also used to identified the tyrosinase inhibitor compound as caffeine (272). 
TABLE 5 | Purification and identification of the peptides from bee products.

\begin{tabular}{|c|c|c|c|c|c|}
\hline Bee products & Protein/peptide & Molecular weight (kDa) & Separation/purification & Identification/analysis & References \\
\hline RJ & $\begin{array}{l}\text { MRJP1 [A. mellifera } \\
\text { MRJP (AmMRJP)] } \\
\text { AmMRJPs 1-7 }\end{array}$ & & $\begin{array}{l}\text { The MagneHis }{ }^{T M} \text { Protein } \\
\text { Purification System (Promega, } \\
\text { Madison, WI, USA) }\end{array}$ & $\begin{array}{l}\text { SDS-PAGE and western } \\
\text { blot analysis }\end{array}$ & $(19,201)$ \\
\hline RJ (China) & MRJP1-3 & $\begin{array}{l}228 \text { kDa MRJP1 oligomer } 1 \text {; } \\
408 \text { kDa MRJP1 oligomer } 2 \text { a; } \\
639 \text { kDa MRJP1 oligomer } 3\end{array}$ & $\begin{array}{l}\text { Hydrophobic interaction } \\
\text { chromatography; } \\
\text { lon-exchange chromatography, } \\
\text { Size exclusion chromatography }\end{array}$ & $\begin{array}{l}\text { Native-PAGE (SDS-PAGE) } \\
\text { LC-MS/MS (SRCD) } \\
\text { spectroscopy (SEC-HPLC) }\end{array}$ & (209) \\
\hline $\begin{array}{l}\text { Black Dwarf } \\
\text { Honey and BCP } \\
\text { (Thailand) }\end{array}$ & & & & $\begin{array}{l}\text { ARACUS amino acid } \\
\text { analyzer; } \\
\text { high-performance liquid } \\
\text { chromatography }\end{array}$ & (203) \\
\hline RJ & $\begin{array}{l}\text { Royal actin } \\
\text { MRJP1 oligomer }\end{array}$ & $\begin{array}{l}55 \mathrm{kDa} \\
280 \mathrm{kDa}\end{array}$ & & & (218) \\
\hline RJ & MRJP1 oligomer & $350 \mathrm{kDa}$ & & & (219) \\
\hline RJ (Japan) & MRJP1 oligomer & $420 \mathrm{kDa}$ & $\begin{array}{l}\text { Size-exclusion and } \\
\text { anion-exchange HPLC }\end{array}$ & $\begin{array}{l}\text { 2-D blue } \\
\text { native/SDS-PAGE. } \\
\text { MALDI-TOF-MS } \\
\text { SDS-PAGE } \\
\text { Blue native-PAGE }\end{array}$ & (204) \\
\hline RJ (Japan) & $\begin{array}{l}\text { MRJP2: Royal jelly } \\
\text { glycol-protein }\end{array}$ & $55 \mathrm{kDa}$ & Cosmosil 5Cl8-AR Column & $\begin{array}{l}\text { RP-HPLC } \\
\text { MALDI-TOF MS }\end{array}$ & (220) \\
\hline RJ (Japan) & $\begin{array}{l}\text { MRJP2 and MRJP3 } \\
\text { MRJP1 }\end{array}$ & $\begin{array}{l}(52 \mathrm{kDa}) \\
(60-70 \mathrm{kDa}) \\
(290 \mathrm{kDa})\end{array}$ & & $\begin{array}{l}\text { Size-exclusion HPLC, } \\
\text { SDS-PAGE and 2-DE } \\
\text { Superose } 12 \text { HPLC } \\
\text { SDS-PAGE }\end{array}$ & (195) \\
\hline RJ & $\begin{array}{l}\text { RJP-1 } \\
\text { RJP57-1 }\end{array}$ & & & & (221) \\
\hline RJ (China) & MRJP5 & $80 \mathrm{kDa}$ & & $\begin{array}{l}\text { 2D-PAGE } \\
\text { (MALDI-TOF/MS) }\end{array}$ & (214) \\
\hline RJ (Japan) & & $55 \mathrm{kDa}$ & $\begin{array}{l}\text { Gel filtration on Sephadex G-100; } \\
\text { Reverse-phase HPLC; Gel } \\
\text { filtration on a GCL-90 column }\end{array}$ & SDS-PAGE & (196) \\
\hline RJ (China) & $\begin{array}{l}\text { MRJP1, MRJP2, } \\
\text { and MRJP3 }\end{array}$ & $\begin{array}{l}48.6 \mathrm{kDa} \\
45.06 \mathrm{kDa} \\
46.27 \mathrm{kDa}\end{array}$ & $\begin{array}{l}\text { Extracted and separated using } \\
\text { two-dimensional electrophoresis } \\
(2-D E)\end{array}$ & $\begin{array}{l}\text { (SDS-PAGE) } \\
\text { (MALDI-TOF/MS) (Bruker } \\
\text { Daltonics) } \\
\text { LC-Chip/ESI-QTOF-MS }\end{array}$ & (80) \\
\hline BV (Egypt) & $\begin{array}{l}\text { Melittin } \\
\text { Apamin } \\
\text { MCD peptide } \\
\text { Adolapin } \\
\text { Minimine } \\
\text { Procamine A, B } \\
\text { Secarpin } \\
\text { Tertiapin } \\
\text { Melittin F }\end{array}$ & $\begin{array}{l}2,840 \\
2,036 \\
2,588 \\
11,500 \\
6,000 \\
500 \\
2,600 \\
2,000 \\
2,840\end{array}$ & & & (222) \\
\hline
\end{tabular}


TABLE 5 | Continued

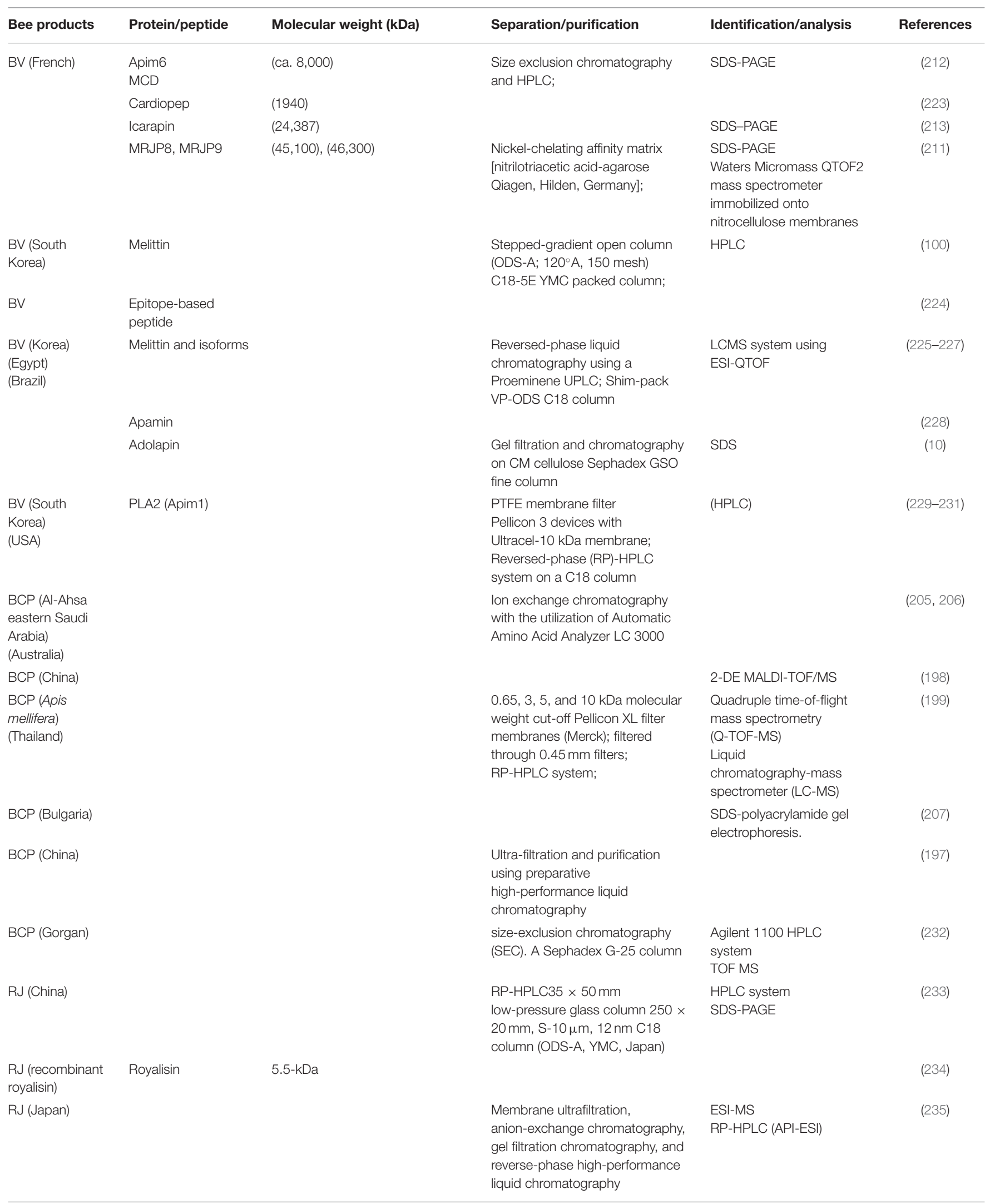


TABLE 5 | Continued

\begin{tabular}{|c|c|c|c|c|c|}
\hline Bee products & Protein/peptide & Molecular weight (kDa) & Separation/purification & Identification/analysis & References \\
\hline BCP (China) & Polypeptide-2 & $42,388 \mathrm{Da}$ & $\begin{array}{l}\text { Ion-exchange column and gel } \\
\text { filtration chromatography }\end{array}$ & $\begin{array}{l}\text { MALDI-TOF-MS, LC-MS } \\
\text { immunological histological } \\
\text { chemistry transmission } \\
\text { electron microscope } \\
\text { SDS-PAGE }\end{array}$ & (215) \\
\hline
\end{tabular}

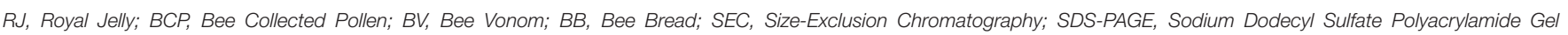

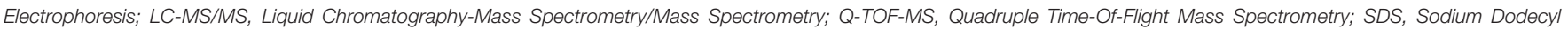

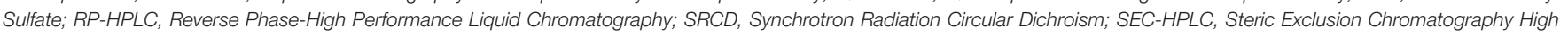

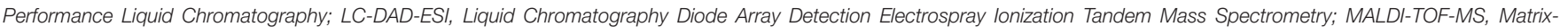

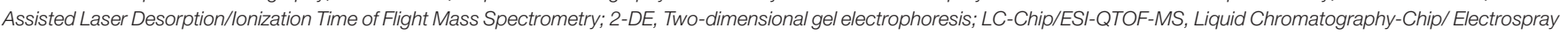
Ionization-Quadruple Time-of-Flight Mass Spectrometry.

\section{Summary}

- Drying techniques are essential for the storage of the bee products;

- Traditional drying methods include freezing, solar drying and in a hot air chamber. Hot air drying has a reasonably shorter processing time, the most adequate temperature for BCP drying is at $60^{\circ} \mathrm{C}$, but hot air drying has an effect on the $\mathrm{BCP}$ quality and organoleptic characteristics, physicochemical properties, morphological structure; FD is considered the most gentle drying method, with fast rehydration rates and high rehydration capacity;

- The freezing and FD methods are used to preserve RJ, however some researchers pointed that FD RJ is highly susceptible to Maillard reaction (MR) and cannot meet the product quality and stability requirements;

- Innovative drying methods like IR, thin-layer IR, microwave, microwave-assisted vacuum drying (MW-VD), low temperature high velocity (LTHV)-assisted fluidized bed drying have been applied in the BCP drying;

- Several biotechnological solutions: fermentation with the yeast and enzyme hydrolysis; physical processing technology: ultrasonication and high shear (US-HS) technique, ultrasonic temperature difference, ultrasonic and ball-milling treatment, supercritical carbon dioxide $\left(\mathrm{CO}_{2}\right)$ techniques have been used in the wall breaking;

\section{PROCESSING TECHNIQUES APPLIED IN THE BEE PRODUCTS QUALITY CONTROL}

\section{Assessment of the Bee Products}

Among all the methods, morphometric analysis for morphology or surface texture and molecular structural analysis were the two aspects mainly for the differentiation or identification of compounds from bee products. The assessment methods applied in the bee products are shown in Table 7 .

Microscopic (SEM) and spectroscopic methods (FT Raman; FT-MIR-ATR; FT-NIR; and Vis) were used to identify pollen grains and interpret the specific contribution of the main chemical constituents (126). SEM usually analyzes the morphometry and surface of the BCP (276) and the morphological changes after treatment (148). GC and GC-MS techniques were used to identify and confirm free amino acids in RJ (277) or the fatty acids in the bee products (112). Quick, easy, and non-destructive tools are used to ensure the quality and efficacy of PP and CP for clinical therapy $(273,278)$. FT-IR and 2D-IR spectroscopy were used to identify the Cattail pollen (CP) and pine pollen (PP) from BCP. In addition, differential scanning calorimetry (DSC) and FTIR spectroscopy were used to study the influence of aging conditions (274) and verify the authenticity of the beeswax (279). Matrix-assisted laser desorption/ionization mass spectrometry (MALDI-MS) was used to detect honey adulteration based on oligosaccharide and polysaccharide profiles (134).

Recently, omics technologies have been applied in honeybee product research to assess authentication, adulteration detection, quality, safety, and traceability of bee products, bioactivity (antimicrobial and antioxidant), microbiome characterization, and human health effects (280).

Electrochemical techniques (sensors and biosensors) are the predominant methodology that can be applied for the quantification of individual or total phenolic compounds, either in standard solutions or in real matrices (e.g., plants, fruits, and beverages) and their capability for assessing antioxidant activity/capacity (281). An electrochemical sensing platform based on a silver ion-cross-linked hydrogel for the evaluation of three different bee products has also been proposed (39). Electrochemical techniques have been acted as essential analytical tools for the characterization especial the antioxidant properties of the honey and propolis samples in an early stage (281).

\section{Bee Products Quality Control (Techniques and Standard) \\ Water Content and Microbial Quality Control}

The moisture content and microbiological safety are the two key parameters to control to ensure the quality of bee products, especially for $\mathrm{BCP}$ and $\mathrm{BB}$. Therefore, some standards or technologies are emerging at the same time. The presence of microorganisms in honey is an important indicator of quality. The drying out process for the bee product should ensure that the temperature lower than $42^{\circ} \mathrm{C}$, with a water content $<6 \%$ (282, 283). Minimal requirements for dried BCP have been established in Brazil: max. 4\%; Switzerland and Poland: max. 6\%; Uruguay: max. 8\%; Bulgaria: max. 10\% (283). The bacterial load should be control within legal hygienic limits (68). In addition, destruction 
TABLE 6 | Peptides sequence and bioactivity from bee products.

\begin{tabular}{|c|c|c|c|c|}
\hline Bee products & Peptides & Sequence and structure & Bioactivity & References \\
\hline & $\begin{array}{l}\text { MRJP2 } \\
49 \mathrm{kDa}\end{array}$ & AIVRENSPRNLEK & & \\
\hline & $\begin{array}{l}\text { MRJP3 } \\
60-70 \mathrm{kDa}\end{array}$ & AAVNHQ (R/K)KSANNLAHS & & \\
\hline \multirow[t]{2}{*}{ BCP (Thailand) } & $\mathrm{H} 2$ & $\begin{array}{l}\text { VLAKNAPP } \\
\text { RLNTAEAGH }\end{array}$ & $\begin{array}{l}\text { NO scavenging activity } \\
I_{50}: 3.55 \pm 0.09 \mathrm{mg} / \mathrm{mL}\end{array}$ & (199) \\
\hline & $\mathrm{H} 3$ & $\begin{array}{l}\text { VTAHSATVLPR } \\
\text { KNKKWPAEAAH } \\
\text { KLRSRNLLHPT } \\
\text { TNRLLSGHSAKKH }\end{array}$ & $1.57 \pm 0.11$ & \\
\hline \multirow[t]{22}{*}{ RJ (Japan) } & $\mathrm{F} 1-1$ & IA & & (235) \\
\hline & $\mathrm{F} 1-2$ & LT & & \\
\hline & $\mathrm{F} 1-3$ & ATA & & \\
\hline & $\mathrm{F} 1-4$ & AT & & \\
\hline & F1-5 & $\mathrm{PL}$ & & \\
\hline & F1-6 & $\mathrm{PL}$ & & \\
\hline & $\mathrm{F} 1-7$ & LP & & \\
\hline & $\mathrm{F} 1-8$ & LASTP & & \\
\hline & $\mathrm{F} 4-1$ & TGG & & \\
\hline & F4-6 & GIPHA & & \\
\hline & F4-11 & IGIPHA & & \\
\hline & $\mathrm{F} 2-1$ & LAA & & \\
\hline & F6-2 & PAA & & \\
\hline & F6-4 & TAT & & \\
\hline & F5-12 & GIPHA & & \\
\hline & F5-13 & TAGH & & \\
\hline & F5-15 & $\pi$ & & \\
\hline & F5-7 & VATGG & & \\
\hline & F5-9 & IAGGS & & \\
\hline & $\mathrm{F} 5-10$ & IAGG & & \\
\hline & $\mathrm{Fd}-1$ & $\mathrm{LL}$ & & \\
\hline & $\mathrm{Fd}-2$ & HGT & & \\
\hline \multirow[t]{11}{*}{ RJ (synthesis) } & RJI & PFKISIHL & & (236) \\
\hline & RJ ॥ & TPFKISIHL & & \\
\hline & RJ III & EPFKISIHL & & \\
\hline & RJ IC & PFKISIHLGGY & & \\
\hline & RJ $\| C$ & TPFKISIHLGGY & & \\
\hline & RJ IIC & EPFKISIHLGGY & & \\
\hline & RJIN & YGGPFKISIHL & & \\
\hline & RJ IIN & YGGTPFKISIHL & & \\
\hline & RJ IIN & YGGEPFKISIHL & & \\
\hline & TA & FLPLIGRVLSGIL & & \\
\hline & TB & LLPI VGNLLKSLL & & \\
\hline
\end{tabular}

ACE, Angiotensin-I Converting Enzyme; $I_{50}$, half maximal Inhibitory Concentration; BCP, Bee Collected Pollen; RJ, Royal Jelly. 
TABLE 7 | Assessment methods for the bee products.

\begin{tabular}{|c|c|c|c|}
\hline Bee products & Techniques & Functions & References \\
\hline RJ (Turkey) & FTIR & $\begin{array}{l}\diamond \text { Prediction of the royal jelly content in } \\
\text { hive products }\end{array}$ & $(273)$ \\
\hline BB (Ukraine) & $\begin{array}{l}\text { Microscopic (SEM) and spectroscopic } \\
\text { FT Raman and Vis, FT-MIR ATR, FT-NIR }\end{array}$ & $\begin{array}{l}\diamond \text { Discrimination of bee bread samples } \\
\text { Morphometric analysis }\end{array}$ & $(126)$ \\
\hline BCP (Turkey) & SEM & $\diamond$ Morphological analysis & $(148)$ \\
\hline Beeswax (Slovakia) & $\begin{array}{l}\text { FTIR } \\
\text { Differential scanning calorimetry }\end{array}$ & & $(274)$ \\
\hline BCP (China) & SEM, TEM, Particle size distribution & $\diamond$ Morphological observation & $(162)$ \\
\hline Rape BCP (China) & SEM & & $(156)$ \\
\hline Honey (China) & MALDI-MS & $\begin{array}{l}\diamond \text { Detection of honey adulteration based } \\
\text { on oligosaccharide and polysaccharide } \\
\text { profiles }\end{array}$ & $(134)$ \\
\hline BCP (Turkey) & $\begin{array}{l}\text { HP-TLC } \\
\text { HPLC }\end{array}$ & $\begin{array}{l}\diamond \text { Fingerprinting of the phenolic } \\
\text { compounds }\end{array}$ & $(275)$ \\
\hline BCP, propolis (Turkey) & GC-MS, SEM & $\begin{array}{l}\diamond \text { Analysis of the chemical composition } \\
\diamond \text { Evaluation of the shape and size }\end{array}$ & $(269)$ \\
\hline
\end{tabular}

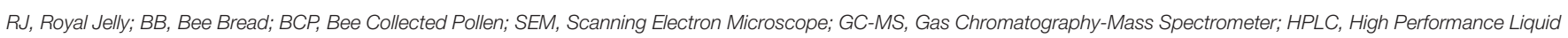

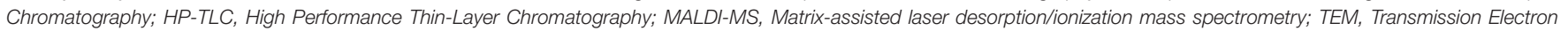
Microscope; FT-MIR, Fourier Transform micro infrared spectroscopy, FT-NIR, Fourier Transform Near infrared spectroscopy; FTIR, Fourier Transform infrared spectroscopy.

of bacteria using chemical fumigants (284) or irradiation, ozone treatments (285) is not necessary and leads to toxic residues, but can control the microbiological quality (285). The European Union make the standard for microbiological quality follows AOAC methods and levels (283). While the major taxon needs to be not $<80 \%$ (283).

\section{Contaminant Control}

BCP is least influenced by contaminants from beekeeping. The main contaminants in BCP are the heavy metals (286) and pesticides (287) originating from the environment and agricultural practices. BCP should gather in the areas at least $3 \mathrm{~km}$ from the contamination sources for control the quality. Requirements for heavy metal content of BCP loads are no more than: $\mathrm{Cd} ; 1 \mathrm{mg} / 10 \mathrm{~kg}, \mathrm{~Pb} ; 5 \mathrm{mg} / 10 \mathrm{~kg}, \mathrm{As} ; \mathrm{mg} / 10 \mathrm{~kg}$, and $\mathrm{Hg} ; 0.3$ $\mathrm{mg} / 10 \mathrm{~kg}$ (283).

The residual levels of three chemicals (i.e., imidacloprid, thiamethoxam, and chlorpyrifos) were higher in pollen than in bee bread (288). BCP from genetically modified organisms (GMOs) have a compulsory requirement (Regulation EC $1829 / 2003)$ to label products where the GMO content exceeds $1 \%$, and the requirement could also be applied to BCP and honey (283). The dominant chemicals were the herbicides atrazine and trifluralin, followed by the insecticide chlorpyrifos thiamethoxam found only in one honey sample $(289,290)$.

\section{Others}

Detecting the compounds in bee products is a good strategy to compare samples from different regions and ensure the quality of bee products. The analysis of polysaccharides is a simple and efficient tool for honey quality control and detection of product adulteration (174). HPLC in gradient mode coupled with photodiode array detection (PAD) method remains for assay the most relevant propolis components. APCI-IT-MS represents a valuable alternative analysis method to obtain typical fingerprints of propolis and can be reliable identify a large number of propolis components (291). A suitable headspace solid-phase micro extraction (HS-SPME) techniques act as new and reliable tools were applied to determine the fingerprint of raw propolis samples from different Italian regions, thus providing the complete chemical characterization of this bioactive material (292). Raman spectroscopy combined with partial least squareslinear discriminant analysis as a potential technique have applied to detect the adulterants (high-fructose corn syrup and maltose syrup) in honey (293).

Moisture, HMF (Hydroxymethyl furfural), free acid, diastase are the composition and quality criteria used routinely for the international honey trade $(135,294)$. Knowledge of the physical or physicochemical properties of bee products is important for monitoring the quality of honey. The hot wire and dynamic plane source methods showed that both methods are suitable for the identification of the bee products thermal parameters (295). Multi-component analysis coupled with modern statistical data evaluation techniques seems to be a promising approach for authenticating honeys.

The nutrient content of the pollen also changes with storage. Thermophysical parameters are substantially correlated with the quality of bee products. Knowledge about the bee products thermophysical characteristics during thermal manipulation can improve the technological processing and storage (295). The color of the honey determined its value for marketing and its end-use. Sensory analysis can also help to determine the botanical origin of the honey (296).

\section{Summary}

Microscopic (SEM) and spectroscopic methods (FT Raman; FTMIR-ATR; FT-NIR; and Vis) were used to identify pollen grains. modern techniques like omics technologies and electrochemical techniques (sensors and biosensors) are the predominant 
methodology to assess authentication, adulteration detection, quality, safety, and traceability of bee products.

\section{CONCLUSIONS AND FUTURE PROSPECTS}

Hive products and the apitherapy have a long history dating back to the old times, which being used in phytotherapy and diet due to their powerful healing properties. Bee products as natural medicines are gaining prominence for the constituent bioactive compounds possess beneficial health properties and are widely used in food, medicines, and cosmetics. Convenient and efficient machines have been designed, and proper processing techniques have been applied based on the properties of the bee products.

Traditional drying techniques such as sun drying, hot air chamber, and FD are used to dehydrate the BCP and BB after harvesting. FD is considered the most effective technique, as it causes negligible damage to bee products; it has been used to dry bee pollen, bee bread, and preserve RJ. But some researchers pointed that RJ samples are not suitable for the FD techniques, for it may cause MR even at lower temperature for some days, the mechanism of that deserve more study. The RJ storage in industrial scales also need more emphasized in the future.

Innovative drying technologies are emerging these years to conserve the good quality of bee products. The water content for the BCP should control $<6$ or $4 \%$ in other country. The traditional drying temperature control at $60^{\circ} \mathrm{C}$ relative better to escape the water and deduce the microbial metabolism. MWD, IR radiation drying, thin-layer IR drying, vacuum drying alone and combined with other methods. MW-VD, LTHV-assisted fluidized bed drying, and IR heating-assisted fluidized bed drying are also widely used. These innovative drying methods can effectively escape the water, but have a marked effect on the quality characteristics, especially color. Beside the water escape effect, drying can also kill microorganisms of the bee products to some extent; irradiation, ozone treatments, and chemical fumigants can also control the microbes in bee products. New cold sterilization technologies should be applied in the future and combined with drying techniques.

Wall-breaking techniques special used for BCP and BB, biotechnological techniques (fermentation with yeast) and physical pretreatments have been used to increase the accessibility of nutrients for intestinal absorption. During fermentation, enzymes that can hydrolyze the wall of the BCPs are produced. The addition of different enzymes, such as pepsin, trypsin, and papain, can also effectively rupture the wall. Physical pretreatment like temperature difference, supercritical $\mathrm{CO}_{2}$, combined ultrasonic-ball-milling treatment, higher shear technique, and temperature difference with ultrasonication showed better wall-breaking efficiency. After fermentation or physical pretreatment, wall-breaking bee pollen yielded better active ingredients with excellent bioactivity. In the further study, wall-breaking technology should focus on the combine treatment, like the physical with fermentation and combine with ultrasound assist enzyme hydrolysis, which can produce the bee products for special patient (higher protein and peptide and less carbohydrate).

Extraction, isolation, and purification techniques are required for almost all bee products to obtain active ingredients. Carbohydrates, proteins, lipids, and fatty acids are the main compounds in BCP, BB, and RJ. Propolis is rich in phenols and flavonoids. The venom contains many peptides such as melittin or isoforms with excellent pharmacological effects. Recently, several active ingredients have been isolated. For the extraction technologies, related studies usually focus on the traditional methods, extraction using water, solvents or solvents with water, and Soxhlet extraction for obtaining oil or fatty acids by using a Soxtec HT 1043 system or solvent extraction alone. Different chromatography columns are widely used for the isolation of each compound, including DEAE cellulose DE-52. They include size-exclusion and anion-exchange HPLC, hydrophobic interaction chromatography, ion-exchange chromatography, size exclusion chromatography, gel-filtration chromatography, and affinity chromatography. Currently, procedures for isolation are tedious and only useful for analytical or preparative purposes in the laboratory, but unsuitable for large-scale processing; thus, new, facile, and efficient technologies should be applied for the extraction and isolation of active ingredients. Highspeed countercurrent chromatography, which is a liquid-liquid separation technology, should be used more for isolating other small active ingredients, such as peptides in the future. Ultrasonication is an innovative technique that can be an efficient technique to assist in the extraction and isolation of lipids or other active ingredients.

For the different techniques, there is still scope for improvement in terms of the characteristics of the samples or the processing steps. Using combined physical processing techniques is a viable way to efficiently obtain more desirable bee products. In addition, to apply each technique on a pilot, or industrial scale is always a tough decision in the future.

\section{AUTHOR CONTRIBUTIONS}

$\mathrm{XL}$ conceived of the presented idea. XZ developed the theory and performed the computations. CG verified the analytical methods. $\mathrm{HM}$ encouraged XL to investigate pollen broken and supervised the findings of this work. All authors discussed the results and contributed to the final manuscript.

\section{SUPPLEMENTARY MATERIAL}

The Supplementary Material for this article can be found online at: https://www.frontiersin.org/articles/10.3389/fnut.2021. 727181/full\#supplementary-material 


\section{REFERENCES}

1. Gupta RK, Stangaciu S. Apitherapy: holistic healing through the honeybee and bee products in countries with poor healthcare system. In: Gupta A, editor. Beekeeping for Poverty Alleviation and Livelihood Security. Springer Netherlands Press (2014). P. 413-46. doi: 10.1007/978-94-017-9199-1_15

2. Gabriele M, Felicioli A, Domenici V, Sagona S, Pucci L, Pozzo L. Phytochemical composition and antioxidant activity of tuscan bee pollen of different botanic origins. Ital J Food Sci. (2015) 27:248-59. doi: 10.14674/1120-1770/ijfs.v191

3. Sattler JAG, de Melo ILP, Granato D, Araújo E, da Silva de Freitas A, Barth OM, et al. Impact of origin on bioactive compounds and nutritional composition of bee pollen from southern Brazil: a screening study. Food Res Int. (2015) 77:82-91. doi: 10.1016/j.foodres.2015.09.013

4. Lee JD, Park HJ, Chae Y, Lim S. An overview of bee venom acupuncture in the treatment of arthritis. Evid Based Complement Alternative Med eCAM. (2005) 2:79-84. doi: 10.1093/ecam/neh070

5. Adewole AM, Ileke KD, Oluyede PO. Perception and knowledge of bee venom therapy as an alternative treatment for common ailments in southwestern Nigeria. FUTA J Res Sci. (2013) 9:235-40. Available online at: https://www.futa.edu.ng/journal/papers/paper_2_1564057990.pdf

6. Kieliszek M, Piwowarek K, Kot AM, Błazejak S, Chlebowska-Smigiel A, Wolska I. Pollen and bee bread as new health-oriented products: a review. Trends Food Sci Technol. (2018) 71:170-80. doi: 10.1016/j.tifs.2017. 10.021

7. Crane E. "The Quarterly Review of Biology" Bees and Beekeeping: Science, Practice and World Resources. Ithaca, NY: Cornell University Press (1990). p. 614.

8. Ali MAA-SM. Studies on bee venom and its medical uses. Int $J$ Adv Res Technol. (2012) 1:1-15.

9. Bertoncelj J, Polak T, Pucihar T, Lilek N, Kandolf Borovšak A, Korošec M. Carbohydrate composition of Slovenian bee pollens. Int J Food Sci Technol. (2018) 53:1880-8. doi: 10.1111/ijfs.13773

10. Wehbe R, Frangieh J, Rima M, El Obeid D, Sabatier JM, Fajloun Z. Bee venom: overview of main compounds and bioactivities for therapeutic interests. Molecules. (2019) 24:2997. doi: 10.3390/molecules241 62997

11. Ares AM, Valverde S, Bernal JL, Nozal MJ, Bernal J. Extraction and determination of bioactive compounds from bee pollen. $J$ Pharmaceutical Biomed Anal. (2018) 147:110-24. doi: 10.1016/j.jpba.2017. 08.009

12. Li Q-Q, Wang K, Marcucci MC, Sawaya ACHF, Hu L, Xue X-F, et al. Nutrient-rich bee pollen: a treasure trove of active natural metabolites. $J$ Funct Foods. (2018) 49:472-84. doi: 10.1016/j.jff.2018.09.008

13. Melliou E, Chinou I. Chemistry and bioactivities of royal jelly. St in Nat Prod Chem. (2014) 43:261-90. doi: 10.1016/B978-0-444-63430-6.00008-4

14. Pohl P, Dzimitrowicz A, Greda K, Jamroz P, Lesniewicz A, SzymczychaMadeja A, et al. Element analysis of bee-collected pollen and bee bread by atomic and mass spectrometry - methodological development in addition to environmental and nutritional aspects. TrAC Trends Anal Chem. (2020) 128:115922. doi: 10.1016/j.trac.2020.115922

15. Hernández Zarate MS, Abraham Juárez MdR, Cerón García A, Ozuna López C, Gutiérrez Chávez AJ, Segoviano Garfias JdJN, et al. Flavonoids, phenolic content, and antioxidant activity of propolis from various areas of Guanajuato, Mexico. Food Sci Technol. (2018) 38:210-5. doi: $10.1590 /$ fst. 29916

16. Cornara L, Biagi M, Xiao J, Burlando B. Therapeutic properties of bioactive compounds from different honeybee products. Front Pharmacol. (2017) 8:412. doi: $10.3389 /$ fphar.2017.00412

17. Kocot J, Kielczykowska M, Luchowska-Kocot D, Kurzepa J, Musik I. Antioxidant potential of propolis, bee pollen, and royal jelly: possible medical application. Oxid Med Cell Longev. (2018) 2018:7074209. doi: $10.1155 / 2018 / 7074209$

18. Fratini F, Cilia G, Turchi B, Felicioli A. Beeswax: a minireview of its antimicrobial activity and its application in medicine. Asian Pac J Trop Med. (2016) 9:839-43. doi: 10.1016/j.apjtm.2016.07.003

19. Park HG, Kim BY, Park MJ, Deng Y, Choi YS, Lee KS, et al. Antibacterial activity of major royal jelly proteins of the honeybee
(Apis mellifera) royal jelly. J Asia Pacific Entomol. (2019) 22:737-41. doi: 10.1016/j.aspen.2019.06.005

20. Zhou W, Zhao Y, Yan Y, Mi J, Lu L, Luo Q, et al. Antioxidant and immunomodulatory activities in vitro of polysaccharides from bee collected pollen of Chinese wolfberry. Int J Biol Macromol. (2020) 163:190-9. doi: 10.1016/j.ijbiomac.2020.06.244

21. Banzato TP, Gubiani JR, Bernardi DI, Nogueira CR, Monteiro AF, Juliano FF, et al. Antiproliferative flavanoid dimers isolated from brazilian red propolis. J Natural Products. (2020) 83:1784-93. doi: 10.1021/acs.jnatprod.9b01136

22. Kageyama M, Li K, Sun S, Xing G, Gao R, Lei Z, et al. Antitumor and anti-metastasis activities of honey bee larvae powder by suppressing the expression of EZH2. Biomed Pharmacother. (2018) 105:6906. doi: 10.1016/j.biopha.2018.06.034

23. Maria Campos KRM, Mitchell KA, Proenca da Cunha A. An approach to the characterization of bee pollens via their flavonoid/phenolic profile. Phytochem Anal. (1997) 8:181-5. doi: 10.1002/(SICI)10991565(199707)8:4<181::AID-PCA359>3.0.CO;2-A

24. Chizoba Ekezie F-G, Sun D-W, Han Z, Cheng J-H. Microwave-assisted food processing technologies for enhancing product quality and process efficiency: a review of recent developments. Trends Food Sci Technol. (2017) 67:58-69. doi: 10.1016/j.tifs.2017.05.014

25. Amit SK, Uddin MM, Rahman R, Islam SMR, Khan MS. A review on mechanisms and commercial aspects of food preservation and processing. Agric Food Secur. (2017) 6:51. doi: 10.1186/s40066-017-0130-8

26. Canale A, Benelli G, Castagna A, Sgherri C, Poli P, Serra A, et al. Microwaveassisted drying for the conservation of honeybee pollen. Materials. (2016) 9:363. doi: 10.3390/ma9050363

27. Choi Y-S, Suh H-J, Chung IK. Enhanced extraction of bioactive compounds from bee pollen by wet-grinding technology. J Life Sci. (2016) 26:651-6. doi: $10.5352 /$ JLS.2016.26.6.651

28. Megherbi M, Herbreteau B, Faure R, Dessalces G, Grenier-Loustalot MF. Solid phase extraction of oligo- and polysaccharides; application to maltodextrins and honey qualitative analysis. J Liquid Chromatogr Related Technol. (2008) 31:1033-46. doi: 10.1080/10826070801924915

29. Yang Y, Zhang JL, Zhou Q, Wang L, Huang W, Wang RD. Effect of ultrasonic and ball-milling treatment on cell wall, nutrients, and antioxidant capacity of rose (Rosa rugosa) bee pollen, and identification of bioactive components. $J$ Sci Food Agric. (2019) 99:5350-7. doi: 10.1002/jsfa.9774

30. Bakour M, Fernandes Â, Barros L, Sokovic M, Ferreira ICFR, Badiaa 1. Bee bread as a functional product: chemical composition and bioactive properties. LWT. (2019) 109:276-82. doi: 10.1016/j.lwt.2019.02.008

31. Margaoan R, Strant M, Varadi A, Topal E, Yucel B, Cornea-Cipcigan M, et al. Bee collected pollen and bee bread: bioactive constituents and health benefits. Antioxidants. (2019) 8:568. doi: 10.3390/antiox8120568

32. Abd El-Wahed AA, Khalifa SAM, Sheikh BY, Farag MA, Saeed A, Larik FA, et al. Bee venom composition: from chemistry to biological activity. St Nat Prod Chem. (2019) 60:459-84. doi: 10.1016/B978-0-444-64181-6.00013-9

33. Khalifa SAM, Elashal M, Kieliszek M, Ghazala NE, Farag MA, Saeed A, et al. Recent insights into chemical and pharmacological studies of bee bread. Trends Food Sci Technol. (2020) 97:300-16. doi: 10.1016/j.tifs.2019.08.021

34. Mohammad SM, Mahmud-Ab-Rashid N-K, Zawawi N. Botanical origin and nutritional values of bee bread of stingless bee (Heterotrigona itama) from Malaysia. J Food Qual. (2020) 2020:1-12. doi: 10.1155/2020/2845757

35. Yuan M, Yuan XJ, Pineda M, Liang ZY, He J, Sun SW, et al. A comparative study between Chinese propolis and Brazilian green propolis: metabolite profile and bioactivity. Food Funct. (2020) 11:2368-79. doi: 10.1039/C9FO02051A

36. Omar WAW, Yahaya N, Ghaffar ZA, Fadzilah NH. GC-MS analysis of chemical constituents in ethanolic bee pollen extracts from three species of malaysian stingless bee. J Apicultural Sci. (2018) 62:275-84. doi: 10.2478/jas-2018-0022

37. Gomez-Caravaca AM, Gomez-Romero M, Arraez-Roman D, SeguraCarretero A, Fernandez-Gutierrez A. Advances in the analysis of phenolic compounds in products derived from bees. J Pharmaceutical Biomed Anal. (2006) 41:1220-34. doi: 10.1016/j.jpba.2006.03.002

38. Riddiford LM, Hiruma K, Lan Q, Zhou B. Regulation and role of nuclear receptors during larval molting and metamorphosis of lepidoptera. Am Zool. (1999) 39:736-46. doi: 10.1093/icb/39.4.736 
39. Zheng Y. Electrochemical determination of antioxidant activity of different bee products. Int J Electrochem Sci. (2019) 14:3663-72. doi: 10.20964/2019.04.09

40. Pasupuleti VR, Sammugam L, Ramesh N, Gan SH. Honey, propolis, and royal jelly: a comprehensive review of their biological actions and health benefits. Oxidative Med Cell Longevity. (2017) 2017:1259510. doi: $10.1155 / 2017 / 1259510$

41. Samarghandian S, Farkhondeh T, Samini F. Honey and health: a review of recent clinical research. Pharmacognosy Res. (2017) 9:121-7. doi: 10.4103/0974-8490.204647

42. Adebolu TT. Effect of natural honey on local isolates of diarrhea causing bacteria in southwestern Nigeria. Afr J Biotechnol. (2005) 4:1172-4. doi: 10.5897/AJB2005.000-3233

43. Nipun Dashora VS, Jaykumar Bhagat, Prabhu KS, Lobo R. Antitumor activity of dendrophthoe falcata against ehrlich ascites carcinoma in Swiss Albino Mice. Pharmaceutical Crops. (2011) 2:1-7. doi: 10.2174/2210290601102010001

44. Bergman A, Yanai J, Weiss J, Bell D, David MP. Acceleration of wound healing by topical application of honey: an animal model. Am J Surg. (1983) 145:374-6. doi: 10.1016/0002-9610(83)90204-0

45. Ballantine IT, Khurshid A, Ahsan MM. The Qur'an: basic teachings. Springer Netherlands Press (2015). p. 27-260.

46. Rao PV, Krishnan KT, Salleh N, Gan SH. Biological and therapeutic effects of honey produced by honey bees and stingless bees: a comparative review. Rev Brasil Farmacognosia. (2016) 26:657-64. doi: 10.1016/j.bjp.2016.01.012

47. Lay-flurrie K. Honey in wound care: effects, clinical application and patient benefit. Br J Nurs. (2008) 17:S30-6. doi: 10.12968/bjon.2008.17.Sup5.29649

48. El-Soud NHA. Honey between traditional uses and recent medicine. Macedonian J Med Sci. (2012) 5:205-14. doi: 10.3889/mjms.1857-5773.2012.0213

49. Mijanur Rahman M, Gan SH, Khalil MI. Neurological effects of honey: current and future prospects. Evid Based Complement Alternative Med eCAM. (2014) 2014:958721. doi: 10.1155/2014/958721

50. Ferreres F, García-Viguera C, Tomás-Lorente F, Tomás-Barberán FA. Hesperetin: a marker of the floral origin of citrus honey. J Sci Food Agric. (1993) 61:121-3. doi: 10.1002/jsfa.2740610119

51. Borel LDMS, Marques LG, Prado MM. Performance evaluation of an infrared heating-assisted fluidized bed dryer for processing bee-pollen grains. Chem Eng Process Process Intensification. (2020) 155:108044. doi: 10.1016/j.cep.2020.108044

52. Adaskeviciute V, Kaskoniene V, Kaskonas P, Barcauskaite K, Maruska A. Comparison of physicochemical properties of bee pollen with other bee products. Biomolecules. (2019) 9:819. doi: 10.3390/biom912 0819

53. Duarte AWF, Vasconcelos MRdS, Oda-Souza M, Oliveira FFd, LÓPez AMQ. Honey and bee pollen produced by Meliponini (Apidae) in Alagoas, Brazil: multivariate analysis of physicochemical and antioxidant profiles. Food Sci Technol. (2018) 38:493-503. doi: 10.1590/fst.09317

54. Didaras NA, Karatasou K, Dimitriou TG, Amoutzias GD, Mossialos D. Antimicrobial activity of bee-collected pollen and beebread: state of the art and future perspectives. Antibiotics. (2020) 9:811. doi: 10.3390/antibiotics9110811

55. Estevinho ML, Afonso SE, Feas X. Antifungal effect of lavender honey against Candida albicans, Candida krusei and Cryptococcus neoformans. J Food Sci Technol. (2011) 48:640-3. doi: 10.1007/s13197-011-0243-1

56. Liolios V, Tananaki C, Dimou M, Kanelis D, Rodopoulou MA, Thrasyvoulou A. Exploring the sugar profile of unifloral bee pollen using high performance liquid chromatography. J Food Nutr Res. (2018) 57:1-10.

57. De-Melo AAM, Estevinho LM, Moreira MM, Delerue-Matos C, Freitas AdSd, Barth OM, et al. A multivariate approach based on physicochemical parameters and biological potential for the botanical and geographical discrimination of Brazilian bee pollen. Food Biosci. (2018) 25:91-110. doi: 10.1016/j.fbio.2018.08.001

58. Szabat P, Poleszak J, Szabat M, Boreński S, Wójcik M, Milanowska J. Apitherapy - the medical use of bee products. J Educ Health Sport. (2019) 9:384-96. doi: 10.5281/zenodo.3376968

59. Costa MCA, Morgano MA, Ferreira MMC, Milani RF. Analysis of bee pollen constituents from different Brazilian regions: quantification by NIR spectroscopy and PLS regression. LWT. (2017) 80:76-83. doi: 10.1016/j.lwt.2017.02.003

60. Ryabov EV, Wood GR, Fannon JM, Moore JD, Bull JC, Chandler D, et al. A virulent strain of deformed wing virus (DWV) of honeybees (Apis mellifera) prevails after Varroa destructor-mediated, or in vitro, transmission. PLoS Pathog. (2014) 10:e1004230. doi: 10.1371/journal.ppat.1004230

61. Almeida-Muradian LB, Pamplona LC, Coimbra Sl, Barth OM. Chemical composition and botanical evaluation of dried bee pollen pellets. J Food Composition Anal. (2005) 18:105-11. doi: 10.1016/j.jfca.2003. 10.008

62. Ghosh S, Jung C. Changes in nutritional composition from bee pollen to pollen patty used in bumblebee rearing. J Asia Pacific Entomol. (2020) 23:701-8. doi: 10.1016/j.aspen.2020.04.008

63. Salazar-González C, Díaz-Moreno C. The nutritional and bioactive aptitude of bee pollen for a solid-state fermentation process. J Apicultural Res. (2016) 55:161-75. doi: 10.1080/00218839.2016.1205824

64. Brodschneider R, Crailsheim K. Nutrition and health in honey bees. Apidologie. (2010) 41:278-94. doi: 10.1051/apido/2010012

65. Stanley RG, Linskens HF. Pollen: Biology, Biochemistry, Management. Heidelberg; Berlin; New York, NY: Springer-Verlag (1974).

66. Kostic AZ, Milincic DD, Barac MB, Ali Shariati M, Tesic ZL, Pesic MB. The application of pollen as a functional food and feed ingredient-the present and perspectives. Biomolecules. (2020) 10:84. doi: 10.3390/biom100 10084

67. Campos MGR, Frigerio C, Lopes J, Bogdanov S. What is the future of Bee-Pollen? J ApiProduct ApiMedical Sci. (2010) 2:131-44. doi: 10.3896/IBRA.4.02.4.01

68. Stanley RG, Linskens HF. Pollen: Biology, Biochemistry, Management. Heidelberg; Berlin; New York, NY: Springer-Verlag (1974).

69. Celina Habryka MK, Barbara Drygaś. Bee products used in apitherapy. World Sci News. (2016) 48:254-8. Available online at: https://www.infona.pl/ resource/bwmeta1.element.psid-09a97842-03de-4aa6-8cac-f1bc068a7d8c

70. Martinotti S, Ranzato E. Propolis: a new frontier for wound healing? Burns Trauma. (2015) 3:9. doi: 10.1186/s41038-015-0010-z

71. Yusof N, Abdul Munaim MS, Kutty RV. The effects of different ethanol concentration on total phenolic and total flavonoid content in Malaysian propolis. IOP Confer Ser Mater Sci Eng. (2020) 991:012033. doi: 10.1088/1757-899X/991/1/012033

72. Anjum SI, Ullah A, Khan KA, Attaullah M, Khan H, Ali H, et al. Composition and functional properties of propolis (bee glue): a review. Saudi J Biol Sci. (2019) 26:1695-703. doi: 10.1016/j.sjbs.2018.08.013

73. Wagh VD. Propolis: a wonder bees product and its pharmacological potentials. Adv Pharmacol Sci. (2013) 2013:1-11. doi: 10.1155/2013/308249

74. Veiga RS, De Mendonca S, Mendes PB, Paulino N, Mimica MJ, Lagareiro Netto AA, et al. Artepillin $\mathrm{C}$ and phenolic compounds responsible for antimicrobial and antioxidant activity of green propolis and Baccharis dracunculifolia DC. J Appl Microbiol. (2017) 122:911-20. doi: 10.1111 /jam.13400

75. Vassya Bankova MPaBT. Propolis volatile compounds: chemical diversity and biological activity: a review. Chem Central J. (2014) 8:28. doi: 10.1186/1752-153X-8-28

76. Becerra TB, Calla-Poma RD, Requena-Mendizabal MF, Millones-Gómez PA. Antibacterial effect of peruvian propolis collected during different seasons on the growth of streptococcus mutans. Open Dentistry J. (2019) 13:327-31. doi: $10.2174 / 1874210601913010327$

77. Huang S, Zhang CP, Wang K, Li GQ, Hu FL. Recent advances in the chemical composition of propolis. Molecules. (2014) 19:19610-32. doi: 10.3390/molecules191219610

78. Kosalec I, Bakmaz M, Pepeljnjak S, Vladimir-Kneevi S. Quantitative analysis of the flavonoids in raw propolis from northern Croatia. Acta Pharm. (2004) 54:65-72.

79. Knecht DKH. Patterns of larval food production by hypopharyngeal glands in adult worker honey bees. Apidologie. (1990) 21:457-68. doi: 10.1051/apido:19900507

80. Li J, Feng M, Begna S, Yu F, Zheng A. Proteome comparison of hypopharyngeal gland development between italian and royal jelly producing worker honeybees (Apis mellifera L.). J Proteome Res. (2010) 9:6578-94. doi: 10.1021/pr100768t 
81. Patel NGH, Haydak, MH, Gochnauer TA.Electrophoretic components of the proteins in honeybee larval food. Nature. (1960) 186:633-4. doi: $10.1038 / 186633 \mathrm{a} 0$

82. Ahmad S, Campos MG, Fratini F, Altaye SZ, Li J. New insights into the biological and pharmaceutical properties of royal jelly. Int J Mol Sci. (2020) 21:382. doi: $10.3390 / \mathrm{ijms} 21020382$

83. Hu F-L, Bíliková K, Casabianca H, Daniele G, Salmen Espindola F, Feng M, et al. Standard methods for Apis mellifera royal jelly research. J Apicultural Res. (2017) 58:1-68. doi: 10.1080/00218839.2017.1286003

84. Altaye SZ, Meng L, Li J. Molecular insights into the enhanced performance of royal jelly secretion by a stock of honeybee (Apis mellifera ligustica) selected for increasing royal jelly production. Apidologie. (2019) 50:436-53. doi: 10.1007/s13592-019-00656-1

85. Feng M, Fang Y, Han B, Xu X, Fan P, Hao Y, et al. In-depth N-glycosylation reveals species-specific modifications and functions of the royal jelly protein from western (Apis mellifera) and Eastern Honeybees (Apis cerana). J Proteome Res. (2015) 14:5327-40. doi: 10.1021/acs.jproteome.5b00829

86. Maghsoudlou A, Mahoonak AS, Mohebodini H, Toldra F. Royal jelly: chemistry, storage and bioactivities. J Apicultural Sci. (2019) 63:17-40. doi: 10.2478/jas-2019-0007

87. Wang Y, Ma L, Zhang W, Cui X, Wang H, Xu B. Comparison of the nutrient composition of royal jelly and worker jelly of honey bees (Apis mellifera). Apidologie. (2015) 47:48-56. doi: 10.1007/s13592-015-0374-x

88. Ramadan MF, Al-Ghamdi A. Bioactive compounds and health-promoting properties of royal jelly: a review. J Funct Foods. (2012) 4:39-52. doi: 10.1016/j.jff.2011.12.007

89. Wang B, Sheng H, Shi Y, Hu W, Hong N, Zeng W, et al. Recent advances for microencapsulation of flame retardant. Polymer Degradation Stability. (2015) 113:96-109. doi: 10.1016/j.polymdegradstab.2015.01.008

90. Stacey RJ. The composition of some Roman medicines: evidence for Pliny's Punic wax? Anal Bioanal Chem. (2011) 401:1749-59. doi: 10.1007/s00216-011-5160-7

91. Patwardhan B. Bridging Ayurveda with evidence-based scientific approaches in medicine. Patwardhan EPMA J. (2014) 5:19. doi: 10.1186/1878-5085-5-19

92. Yusop SATW, Sukairi AH, Sabri WMAW, Asaruddin MR. Antioxidant, antimicrobial and cytotoxicity activities of propolis from beladin, sarawak stingless bees trigona itama extract. Mater Today Proc. (2019) 19:1752-60. doi: 10.1016/j.matpr.2019.11.213

93. Buchwald R, Breed MD, Greenberg AR. The thermal properties of beeswaxes: unexpected findings. J Exp Biol. (2008) 211(Pt 1):121-7. doi: $10.1242 /$ jeb.007583

94. Shaltouki P. Synthesis and characterization of nanoparticles propolis using beeswax. Iran J Chem Chem Eng. (2019) 38:9-19.

95. Ranjha NM, Khan H, Naseem S. Encapsulation and characterization of controlled release flurbiprofen loaded microspheres using beeswax as an encapsulating agent. Journal of materials science Materials in medicine. (2010) 21:1621-30. doi: 10.1007/s10856-010-4034-4

96. Hellner M, Winter D, von Georgi R, Munstedt K. Apitherapy: usage and experience in german beekeepers. Evid Based Complement Alternat Med. (2008) 5:475-9. doi: 10.1093/ecam/nem052

97. Zurier RB, Mitnick H, Bloomgarden D, Weissmann G. Effect of bee venom on experimental arthritis. Ann rheum Dis. (1973) 32:466. doi: 10.1136/ard.32.5.466

98. Zhang S, Liu Y, Ye Y, Wang XR, Lin LT, Xiao LY, et al. Bee venom therapy: potential mechanisms and therapeutic applications. Toxicon. (2018) 148:6473. doi: 10.1016/j.toxicon.2018.04.012

99. Son DJ, Lee JW, Lee YH, Song HS, Lee CK, Hong JT. Therapeutic application of anti-arthritis, pain-releasing, and anti-cancer effects of bee venom and its constituent compounds. Pharmacol Ther. (2007) 115:246-70. doi: $10.1016 /$ j.pharmthera.2007.04.004

100. Lee Y, Kim SG, Kim IS, Lee HD. Standardization of the manufacturing process of bee venom pharmacopuncture containing melittin as the active ingredient. Evid Based Complement Alternat Med. (2018) 2018:2353280. doi: 10.1155/2018/2353280

101. Haber M, Mishyna M, Itzhak Martinez JJ, Benjamin O. Edible larvae and pupae of honey bee (Apis mellifera): odor and nutritional characterization as a function of diet. Food Chem. (2019) 292:197-203. doi: 10.1016/j.foodchem.2019.04.041
102. Jin R, Lin ZJ, Xue CM, Zhang B. An improved association-mining research for exploring Chinese herbal property theory: based on data of the Shennong's Classic of Materia Medica. J Integr Med. (2013) 11:352-65. doi: 10.3736/jintegrmed2013051

103. Li K, Sun S, Kageyama M, Xiao L, Xing G, Gao R, et al. Evaluation of the immunomodulatory and anti-inflammatory activities of honey bee larva powder. J Med Food. (2020) 23:772-82. doi: 10.1089/jmf.2019.4554

104. Ramos-Elorduy J, Moreno JM, Prado EE, Perez MA, Otero JL, De Guevara OL. Nutritional value of edible insects from the state of Oaxaca, Mexico. $J$ Food Composition Anal. (1997) 10:142-57. doi: 10.1006/jfca.1997.0530

105. Finke MD. Nutrient composition of bee brood and its potential as human food. Ecol Food Nutr. (2005) 44:257-70. doi: 10.1080/03670240500187278

106. Ghosh S, Jung C, Meyer-Rochow VB. Nutritional value and chemical composition of larvae, pupae, and adults of worker honey bee, Apis mellifera ligustica as a sustainable food source. J Asia Pacific Entomol. (2016) 19:48795. doi: 10.1016/j.aspen.2016.03.008

107. Dong D, Dong M, Liu K, Lu Y, Yu B. Antioxidant activity of queen bee larvae processed by enzymatic hydrolysis. J Food Process Preserv. (2017) 42:e13461. doi: $10.1111 /$ jfpp. 13461

108. Kageyama M, Xing G, Li K, Zhang Z, Sugiyama A. Oral administration of freeze-dried powders of honey bee larvae inhibits the development of atopic dermatitis-like skin lesions in NC/Nga mice. Personalized Med Univ. (2017) 6:22-7. doi: 10.1016/j.pmu.2017.05.001

109. Melo ILPd, Almeida-Muradian LBd. Stability of antioxidants vitamins in bee pollen samples. Química Nova. (2010) 33:514-8. doi: 10.1590/S0100-40422010000300004

110. Anjos O, Paula V, Delgado T, Estevinho L. Influence of the storage conditions on the quality of bee pollen. Zemdirbyste Agric. (2019) 106:87-94. doi: 10.13080/z-a.2019.106.012

111. Thakur M, Nanda V. Assessment of physico-chemical properties, fatty acid, amino acid and mineral profile of bee pollen from India with a multivariate perspective. J Food Nutr Res. (2018) 57:328-40.

112. Kostić AŽ, Pešić MB, Trbović D, Petronijević R, Dramićanin AM, Milojković-Opsenica DM, et al. The fatty acid profile of Serbian bee-collected pollen - a chemotaxonomic and nutritional approach. J Apicultural Res. (2017) 56:533-42. doi: 10.1080/00218839.2017.1356206

113. Mayda N, Özkök A, Ecem Bayram N, Gerçek YC, Sorkun K. Bee bread and bee pollen of different plant sources: determination of phenolic content, antioxidant activity, fatty acid and element profiles. J Food Measure Characterization. (2020) 14:1795-809. doi: 10.1007/s11694-020-0 0427-y

114. Özcan MM, Aljuhaimi F, Babiker EE, Uslu N, Ceylan DA, Ghafoor K, et al. Determination of antioxidant activity, phenolic compound, mineral contents and fatty acid compositions of bee pollen grains collected from different locations. J Apicultural Sci. (2019) 63:69-79. doi: 10.2478/jas-2019-0004

115. Kayacan S, Sagdic O, Doymaz I. Effects of hot-air and vacuum drying on drying kinetics, bioactive compounds and color of bee pollen. J Food Measure Characterization. (2018) 12:1274-83. doi: 10.1007/s11694-018-9741-4

116. Ozkan K, Sagcan N, Ozulku G, Sagdic O, Toker OS, Muz MN. Bioactive and bioaccessibility characteristics of honeybee pollens collected from different regions of Turkey. J Food Measure Characterization. (2017) 12:581-7. doi: 10.1007/s11694-017-9670-7

117. Nicolson SW, Da Silva Das Neves S, Human H, Pirk CWW. Digestibility and nutritional value of fresh and stored pollen for honey bees (Apis mellifera scutellata). J Insect Physiol. (2018) 107:302-8. doi: 10.1016/j.jinsphys.2017.12.008

118. Isik A, Ozdemir M, Doymaz I. Effect of hot air drying on quality characteristics and physicochemical properties of bee pollen. Food Sci Technol. (2019) 39:224-31. doi: 10.1590/fst.02818

119. Bhargava VUEaHR. Chemical analysis and anti-microbial activity of karnataka bee bread of Apis species. World Appl Sci J. (2014) 32:379-85.

120. Zuluaga-Dominguez CM, Quicazan M. Effect of fermentation on structural characteristics and bioactive compounds of bee-pollen based food. $J$ Apicultural Sci. (2019) 63:209-22. doi: 10.2478/jas-2019-0016

121. Araujo JS, Chambo ED, Costa M, Cavalcante da Silva SMP, Lopes de Carvalho CA, L ME. Chemical composition and biological activities of mono- and heterofloral bee pollen of different geographical origins. Int J Mol Sci. (2017) 18:921. doi: 10.3390/ijms18050921 
122. Belina-Aldemita MD, Schreiner M, D'Amico S. Characterization of phenolic compounds and antioxidative potential of pot-pollen produced by stingless bees (Tetragonula biroi Friese) from the Philippines. J Food Biochem. (2020) 44:e13102. doi: 10.1111/jfbc.13102

123. Dinkov D. Bacterial contamination of vacuum stored flower bee pollen. J Microbiol Biotechnol Food Sci. (2018) 7:387-91. doi: 10.15414/jmbfs.2018.7.4.387-391

124. Estevinho LM, Dias T, Anjos O. Influence of the storage conditions (frozen vs dried) in health-related lipid indexes and antioxidants of bee pollen. Euro J Lipid Sci Technol. (2018) 121:1800393. doi: 10.1002/ejlt.201800393

125. Ceksteryte V, Kurtinaitiene B, Jaškune K, Kretavičius J. The influence of storage conditions on invertase, glucose oxidase activity and free acidity of bee bread and bee-collected pollen mixed with honey and vegetable oils. J Apicultural Res. (2020) 59:862-75. doi: 10.1080/00218839.2020.18 04118

126. Bleha R, Shevtsova TS, KruŽík V, Škorpilová T, Salon I, Erban V, et al. Bee breads from two regions of Eastern Ukraine: composition, physical properties and biological activities. Czech J Food Sci. (2019) 37:9-20. doi: 10.17221/201/2018-CJFS

127. Dranca F, Ursachi F, Oroian M. Bee. bread: physicochemical characterization and phenolic content extraction optimization. Foods. (2020) 9:1358. doi: 10.3390/foods 9101358

128. Donadieu Y. La bonne conservation des produits de Ia ruche. Revue Fr Apic Aout-Sept. (1981) 383.

129. Karaali A, Meydanoglu F, Eke D. Studies on composition, freeze-drying and storage of turkish royal jelly. J Apicultural Res. (2015) 27:182-5. doi: 10.1080/00218839.1988.11100799

130. Messia MC, Caboni MF, Marconi E. Storage stability assessment of freezedried royal jelly by furosine determination. J Agric Food Chem. (2005) 53:4440-3. doi: 10.1021/jf0404647

131. Balkanska R, Kashamov B. Composition and physico-chemical properties of lyophilized royal jelly. Uludag Bee Jo. (2011) 11:114-7. Available online at: https://dergipark.org.tr/en/pub/uluaricilik/issue/52028/162330

132. Kanelis D, Tananaki C, Liolios V, Rodopoulou M-A, Goras G, Argena N, et al. Investigating the effect of supplementary feeding on carbohydrate composition and quantity of royal jelly. Open J Appl Sci. (2018) 08:141-9. doi: 10.4236/ojapps.2018.84011

133. Tedesco R, Barbaro E, Zangrando R, Rizzoli A, Malagnini V, Gambaro A, et al. Carbohydrate determination in honey samples by ion chromatographymass spectrometry (HPAEC-MS). Anal Bioanal Chem. (2020) 412:5217-27. doi: 10.1007/s00216-020-02732-3

134. Qu L, Jiang Y, Huang X, Cui M, Ning F, Liu T, et al. High-throughput monitoring of multiclass syrup adulterants in honey based on the oligosaccharide and polysaccharide profiles by MALDI mass spectrometry. J Agric Food Chem. (2019) 67:11256-61. doi: 10.1021/acs.jafc.9b05317

135. Serrano S, Villarejo M, Espejo R, Jodral M. Chemical and physical parameters of Andalusian honey: classification of Citrus and Eucalyptus honeys by discriminant analysis. Food Chem. (2004) 87:619-25. doi: 10.1016/j.foodchem.2004.01.031

136. Zizic JB, Vukovic NL, Jadranin MB, Andelkovic BD, Tesevic VV, Kacaniova $\mathrm{MM}$, et al. Chemical composition, cytotoxic and antioxidative activities of ethanolic extracts of propolis on HCT-116 cell line. J Sci Food Agric. (2013) 93:3001-9. doi: 10.1002/jsfa.6132

137. Gargouri W, Osés SM, Fernández-Muiño MA, Sancho MT, Kechaou N. Evaluation of bioactive compounds and biological activities of Tunisian propolis. LWT. (2019) 111:328-36. doi: 10.1016/j.lwt.2019.05.044

138. Fan D, Wang H-MD, Janaswamy S, Cavender GA. Green physical processing technologies for the improvement of food quality. J Food Qual. (2018) 2018:1-2. doi: 10.1155/2018/6050424

139. Carpena M, Nunez-Estevez B, Soria-Lopez A, Simal-Gandara J. Bee venom: an updating review of its bioactive molecules and its health applications. Nutrients. (2020) 12:3360. doi: 10.3390/nu12113360

140. Kharchenko S, Oskin S, Tsokur D. Modeling of bee-bread drying process. Eng Rural Dev. (2020) 19:445-9. doi: 10.22616/ERDev.2020.19.TF100

141. Duran A, Quicazan M, Zuluaga-Dominguez C. Effect of solar drying process on bioactive compounds and antioxidant activity in vitro of high andean region bee pollen. Chem Eng Trans. (2019) 75:91-6. doi: $10.3303 /$ CET1975016
142. Zuluaga-Domínguez C, Serrato-Bermudez J, Quicazán M. Influence of drying-related operations on microbiological, structural and physicochemical aspects for processing of bee-pollen. Eng Agric Environ Food. (2018) 11:57-64. doi: 10.1016/j.eaef.2018.01.003

143. Kashirin DE, Uspensky IA, Kostenko MY, Rembalovich GK, Danilov KI, et al. Ulyanov Cyclic convective drying of bee pollen. ARPN J Eng Appl Sci. (2019) 14:916-20.

144. Prosapio V, Lopez-Quiroga E. Freeze-drying technology in foods. Foods. (2020) 9:920. doi: 10.3390/foods 9070920

145. Ranieri A, Benelli G, Castagna A, Sgherri C, Signorini F, Bientinesi M, et al. Freeze-drying duration influences the amino acid and rutin content in honeybee-collected chestnut pollen. Saudi J Biol Sci. (2019) 26:252-5. doi: $10.1016 /$ j.sjbs.2017.08.011

146. Dzung NT. Study of determining the technological mode in the freeze drying process of royal jelly in Viet Nam. Carpathian J Food Sci Technol. (2016) 8:47-62. Available online at: http://chimie-biologie.ubm.ro/ carpathian_journal/Vol_8(2)_2016.pdf

147. Dzung NT, Manh LD, Suc NV. Study technological factors effect on the loss of protein, carbohydrate and lipid inside royal jelly in the freeze drying process. Curr Res J Biol Sci. (2015) 7:22-30. doi: 10.19026/crjbs.7.5203

148. Isik A, Ozdemir M, Doymaz I. Infrared drying of bee pollen: effects and impacts on food components. Czech J Food Sci. (2019) 37:69-74. doi: 10.17221/410/2017-CJFS

149. Radoiu M. Microwave drying process scale-up. Chem Eng Process Process Intensification. (2020) 155:108088. doi: 10.1016/j.cep.2020.108088

150. Conte G, Benelli G, Serra A, Signorini F, Bientinesi M, Nicolella C, et al. Lipid characterization of chestnut and willow honeybee-collected pollen: impact of freeze-drying and microwave-assisted drying. J Food Composition Anal. (2017) 55:12-9. doi: 10.1016/j.jfca.2016.11.001

151. Kanar Y, Mazi BG. HMF formation, diastase activity and proline content changes in bee pollen dried by different drying methods. LWT. (2019) 113:108273. doi: 10.1016/j.lwt.2019.108273

152. Isik A, Ozdemir M, Doymaz I. Investigation of microwave drying on quality attributes, sensory properties and surface structure of bee pollen grains by scanning electron microscopy. Brazil J Chem Eng. (2021) 38:177-88. doi: 10.1007/s43153-020-00088-w

153. Kanar Y, Mazi BG. Effect of different drying methods on antioxidant characteristics of bee-pollen. J Food Measure Characterization. (2019) 13:3376-86. doi: 10.1007/s11694-019-00283-5

154. Castagna A, Benelli G, Conte G, Sgherri C, Signorini F, Nicolella C, et al. Drying techniques and storage: do they affect the nutritional value of bee-collected pollen? Molecules. (2020) 25:4925. doi: 10.3390/molecules 25 214925

155. Kilic A. Low temperature and high velocity assisted fluidized bed drying characteristics of bee pollen as bioactive food. J Food Process Eng. (2020) 43:e13439. doi: 10.1111/jfpe.13439

156. Xu X, Sun L, Dong J, Zhang H. Breaking the cells of rape bee pollen and consecutive extraction of functional oil with supercritical carbon dioxide. Innov Food Sci Emerg Technol. (2009) 10:42-6. doi: 10.1016/j.ifset.2008.08.004

157. Chichiriccò G, Pacini E. Cupressus arizonica pollen wall zonation and in vitro hydration. Plant Systematics Evolution. (2007) 270:231-42. doi: 10.1007/s00606-007-0610-6

158. Yan S, Li Q, Xue X, Wang K, Zhao L, Wu L. Analysis of improved nutritional composition of bee pollen (Brassica campestris L.) after different fermentation treatments. Int J Food Sci Technol. (2019) 54:2169-81. doi: 10.1111/ijfs. 14124

159. Filannino P, Di Cagno R, Gambacorta G, Tlais AZA, Cantatore V, Gobbetti $\mathrm{M}$. Volatilome and bioaccessible phenolics profiles in lab-scale fermented bee pollen. Foods. (2021) 10:286. doi: 10.3390/foods10020286

160. Zuluaga-Domínguez C, Castro-Mercado L, Cecilia Quicazán M. Effect of enzymatic hydrolysis on structural characteristics and bioactive composition of bee-pollen. J Food Process Preserv. (2019) 43:e13983. doi: 10.1111/jfpp. 13983

161. Nagai T, Inoue R, Suzuki N, Myoda T, Nagashima T. Antioxidative ability in a linoleic acid oxidation system and scavenging abilities against active oxygen species of enzymatic hydrolysates from pollen Cistus ladaniferus. Int J Mol Med. (2005) 15:259-63. doi: 10.3892/ijmm.15.2.259 
162. Wu W, Qiao J, Xiao X, Kong L, Dong J, Zhang H. In vitro and in vivo digestion comparison of bee pollen with or without wall-disruption. J Sci Food Agric. (2021) 101:2744-55. doi: 10.1002/jsfa.10902

163. Zhao Y, Yan Y, Zhou W, Chen D, Huang K, Yu S, et al. Effects of polysaccharides from bee collected pollen of Chinese wolfberry on immune response and gut microbiota composition in cyclophosphamidetreated mice. J Funct Foods. (2020) 72:104057. doi: 10.1016/j.jff.2020.1 04057

164. Merzlov S, Lomova N, Narizhniy S, Snizhko O, Voroshchuk V. Optimization of technology for shredding the bee pollen. Eastern Euro J Enterprise Technol. (2017) 5:55-60. doi: 10.15587/1729-4061.2017.1 10504

165. Benton AW, Morse RA, Stewart JD. Venom collection from honey bees. Science. (1963) 142:228-30. doi: 10.1126/science.142.3589.228

166. Gunnison AF. An improved method for collecting the liquid fraction of bee venom. J Apicultural Res. (2015) 5:33-6. doi: 10.1080/00218839.1966.11100129

167. Kim DH, Han SM, Keum MC, Lee S, An BK, Lee SR, et al. Evaluation of bee venom as a novel feed additive in fast-growing broilers. Br Poultry Sci. (2018) 59:435-42. doi: 10.1080/00071668.2018.1476675

168. Sobral F, Sampaio A, Falcao S, Queiroz MJ, Calhelha RC, Vilas-Boas M, et al. Chemical characterization, antioxidant, anti-inflammatory and cytotoxic properties of bee venom collected in Northeast Portugal. Food Chem Toxicol. (2016) 94:172-7. doi: 10.1016/j.fct.2016.06.008

169. Brito JCMd, Bastos EMAF, Heneine LGD, Figueiredo KCdS. Fractionation of Apis mellifera venom by means of ultrafiltration: removal of phospholipase A 2. Brazil J Chem Eng. (2018) 35:229-36. doi: 10.1590/0104-6632.20180351s20160171

170. da Silva RI, Brandão ECV, Brito JCMd, Figueiredo KCdS. Analysis of fouling mechanisms and membrane cleaning during ultrafiltration of bee venom. Separation Sci Technol. (2019) 55:988-93. doi: 10.1080/01496395.2019.1577455

171. Ng CY, Mohammad AW, Ng LY, Jahim JM. Membrane fouling mechanisms during ultrafiltration of skimmed coconut milk. J Food Eng. (2014) 142:190200. doi: 10.1016/j.jfoodeng.2014.06.005

172. Wu X-B, Zhang F, Guan C, Pan Q-Z, Zhou L-B, Yan W-Y, et al. A new method of royal jelly harvesting without grafting larvae. Entomol News. (2015) 124:277-81. doi: 10.3157/021.124.0405

173. Pan QZ, Wu XB, Guan C, Zeng ZJ. A new method of queen rearing without grafting larvae. Am Bee J. (2013) 153:1279-80.

174. Megherbi M, Herbreteau B, Faure R, Salvador A. Polysaccharides as a marker for detection of corn sugar syrup addition in honey. J Agric Food Chem. (2009) 57:2105-11. doi: 10.1021/jf803384q

175. Zhu L, Li J, Wei C, Luo T, Deng Z, Fan Y, et al. A polysaccharide from Fagopyrum esculentum Moench bee pollen alleviates microbiota dysbiosis to improve intestinal barrier function in antibiotic-treated mice. Food Funct. (2020) 11:10519-33. doi: 10.1039/D0FO01948H

176. Zhou W, Yan Y, Mi J, Zhang H, Lu L, Luo Q, et al. Simulated digestion and fermentation in vitro by human gut microbiota of polysaccharides from bee collected pollen of chinese wolfberry. J Agric Food Chem. (2018) 66:898-907. doi: 10.1021/acs.jafc.7b05546

177. Li S, Yang G, Yan J, Wu D, Hou Y, Diao Q, et al. Polysaccharide structure and immunological relationships of RG-I pectin from the bee pollen of Nelumbo nucifera. Int J Biol Macromol. (2018) 111:660-6. doi: 10.1016/j.ijbiomac.2018.01.015

178. Li X, Gong H, Yang S, Yang L, Fan Y, Zhou Y. Pectic bee pollen polysaccharide from Rosa rugosa alleviates diet-induced hepatic steatosis and insulin resistance via induction of AMPK/mTOR-mediated autophagy. Molecules. (2017) 22:699. doi: 10.3390/molecules22050699

179. Schievano E, Sbrizza M, Zuccato V, Piana L, Tessari M. NMR carbohydrate profile in tracing acacia honey authenticity. Food Chem. (2020) 309:125788. doi: 10.1016/j.foodchem.2019.125788

180. Miguel MG, Antunes MD, Faleiro ML. Honey as a complementary medicine. Integr Med Insights. (2017) 12:1178633717702869. doi: $10.1177 / 1178633717702869$

181. Martinello M, Mutinelli F. Antioxidant activity in bee products: a review. Antioxidants. (2021) 10:71. doi: 10.3390/antiox10010071
182. Vanderplanck M, Leroy B, Wathelet B, Wattiez R, Michez D. Standardized protocol to evaluate pollen polypeptides as bee food source. Apidologie. (2013) 45:192-204. doi: 10.1007/s13592-013-0239-0

183. Bernal J, Mendiola JA, Ibanez E, Cifuentes A. Advanced analysis of nutraceuticals. J Pharmaceutical Biomed Anal. (2011) 55:758-74. doi: 10.1016/j.jpba.2010.11.033

184. Sagona S, Pozzo L, Peiretti PG, Biondi C, Giusti M, Gabriele M, et al. Palynological origin, chemical composition, lipid peroxidation and fatty acid profile of organic Tuscanian bee-pollen. J Apicultural Res. (2017) 56:136-43. doi: 10.1080/00218839.2017.1287995

185. Li Q, Liang X, Xue X, Wang K, Wu L. Lipidomics provides novel insights into understanding the bee pollen lipids transepithelial transport and metabolism in human intestinal cells. J Agric Food Chem. (2020) 68:907-17. doi: 10.1021/acs.jafc.9b06531

186. Gardana C, Del Bo' C, Quicazán MC, Corrrea AR, Simonetti P. Nutrients, phytochemicals and botanical origin of commercial bee pollen from different geographical areas. J Food Composition Anal. (2018) 73:29-38. doi: 10.1016/j.jfca.2018.07.009

187. Ferioli F, Armaforte E, Caboni MF. Comparison of the lipid content, fatty acid profile and sterol composition in local Italian and commercial royal jelly samples. J Am Oil Chemists' Soc. (2014) 91:875-84. doi: 10.1007/s11746-014-2446-x

188. Kokotou MG, Mantzourani C, Babaiti R, Kokotos G. Study of the royal jelly free fatty acids by liquid chromatography-high resolution mass spectrometry (LC-HRMS). Metabolites. (2020) 10:40. doi: 10.3390/metabo10010040

189. Ache M, Delgado-Raack S, Molina E, Risch R, Rosell-Melé A. Evidence of bee products processing: a functional definition of a specialized type of macrolithic tool. J Arch Sci Rep. (2017) 14:638-50. doi: 10.1016/j.jasrep.2017.06.025

190. Boulanouar B, Guenane H, Sahin B, Öztürk M, Ghareeb MA, da Graça Miguel M. Fatty acid, mineral content and antioxidant activities of Algerian fat bee pollen. Nova Biotechnol Chimica. (2020) 19:208-15. doi: $10.36547 /$ nbc.v19i2.738

191. Wang X, Wang H, Liu Y, You J, Suo Y. Extraction of pollen lipids by SFECO2and determination of free fatty acids by HPLC. Euro J Lipid Sci Technol. (2009) 111:155-63. doi: 10.1002/ejlt.200800054

192. Schmitzova J, Klaudiny J, Albert Š, Schröder W, Schreckengost W, Hanes J, et al. A family of major royal jelly proteins of the honeybee Apis mellifera L. Cell Mol Life Sci. (1998) 54:1020-30. doi: 10.1007/s000180050229

193. Ares AM, Nozal MJ, Bernal J. Extraction, chemical characterization and biological activity determination of broccoli health promoting compounds. J Chromatogr A. (2013) 1313:78-95. doi: 10.1016/j.chroma.2013. 07.051

194. Yang K, Wu D, Ye X, Liu D, Chen J, Sun P. Characterization of chemical composition of bee pollen in China. J Agric Food Chem. (2013) 61:708-18. doi: 10.1021/jf304056b

195. Nozaki R, Tamura S, Ito A, Moriyama T, Yamaguchi K, Kono T. A rapid method to isolate soluble royal jelly proteins. Food Chem. (2012) 134:2332-7. doi: 10.1016/j.foodchem.2012.03.106

196. Fujiwara S, Imai J, Fujiwara M, Yaeshima T, Kawashima T, Kobayashi K. A potent antibacterial protein in royal jelly. Purification and determination of the primary structure of royalisin. J Biol Chem. (1990) 265:11333-7. doi: 10.1016/S0021-9258(19)38596-5

197. Zhu S, Wang S, Wang L, Huang D, Chen S. Identification and characterization of an angiotensin-I converting enzyme inhibitory peptide from enzymatic hydrolysate of rape (Brassica napus L.) bee pollen. LWT. (2021) 147:111502. doi: 10.1016/j.lwt.2021.111502

198. Li J, Chen J, Zhang Z, Pan Y. Proteome analysis of tea pollen (Camellia sinensis) under different storage conditions. J Agric Food Chem. (2008) 56:7535-44. doi: 10.1021/jf800885z

199. Saisavoey T, Sangtanoo P, Chanchao C, Reamtong O, Karnchanatat A. Identification of novel anti-inflammatory peptides from bee pollen (Apis mellifera) hydrolysate in lipopolysaccharide-stimulated RAW264.7 macrophages. J Apicultural Res. (2020) 60:280-9. doi: 10.1080/00218839.2020.1745434

200. Scarselli R, Donadio E, Giuffrida MG, Fortunato D, Conti A, Balestreri E, et al. Towards royal jelly proteome. Proteomics. (2005) 5:769-76. doi: 10.1002/pmic.200401149 
201. Park MJ, Kim BY, Deng Y, Park HG, Choi YS, Lee KS, et al. Antioxidant capacity of major royal jelly proteins of honeybee (Apis mellifera) royal jelly. J Asia Pacific Entomol. (2020) 23:445-8. doi: 10.1016/j.aspen.2020.03.007

202. Al-Kahtani SN, Taha EK, Khan KA, Ansari MJ, Farag SA, Shawer DMB, et al. Effect of harvest season on the nutritional value of bee pollen protein. PLoS ONE. (2020) 15:e0241393. doi: 10.1371/journal.pone.0241393

203. Sommano SR, Bhat FM, Wongkeaw M, Sriwichai T, Sunanta P, Chuttong B, et al. Amino acid profiling and chemometric relations of black dwarf honey and bee pollen. Front Nutr. (2020) 7:558579. doi: 10.3389/fnut.2020.558579

204. Tamura S, Amano S, Kono T, Kondoh J, Yamaguchi K, Kobayashi $\mathrm{S}$, et al. Molecular characteristics and physiological functions of major royal jelly protein 1 oligomer. Proteomics. (2009) 9:5534-43. doi: $10.1002 /$ pmic. 200900541

205. Taha EA, Al-Kahtani S, Taha R. Protein content and amino acids composition of bee-pollens from major floral sources in AlAhsa, eastern Saudi Arabia. Saudi J Biol Sci. (2019) 26:232-7. doi: 10.1016/j.sjbs.2017.06.003

206. Rabie AL, Wells JD, Dent LK. The nitrogen content of pollen protein. $J$ Apicultural Res. (2015) 22:119-23. doi: 10.1080/00218839.1983.11100572

207. Marinova MD, Tchorbanov BP. Preparation of antioxidant enzymatic hydrolysates from honeybee-collected pollen using plant enzymes. Enzyme Res. (2011) 2010:415949. doi: 10.4061/2010/415949

208. Hadi A, Rafie N, Arab A. Bee products consumption and cardiovascular diseases risk factors: a systematic review of interventional studies. Int J Food Properties. (2021) 24:115-28. doi: 10.1080/10942912.2020.1867568

209. Wang X, Dong J, Qiao J, Zhang G, Zhang H. Purification and characteristics of individual major royal jelly protein 1-3. J Apicultural Res. (2020) 59:104960. doi: 10.1080/00218839.2020.1761071

210. Šimúth J, Bíliková K, Kováčová E. Royal jelly proteins as a tool for development of functional ingredients for health. Standing Commission Apither. (2003) 2017:42-6. Available online at: http://www.fiitea.org/ foundation/files/312.pdf

211. Blank S, Bantleon FI, McIntyre M, Ollert M, Spillner E. The major royal jelly proteins 8 and 9 (Api m 11) are glycosylated components of Apis mellifera venom with allergenic potential beyond carbohydrate-based reactivity. Clin Exp Allergy. (2012) 42:976-85. doi: 10.1111/j.1365-2222.2012.03966.x

212. Kettner A, Hughes GJ, Frutiger S, Astori M, Roggero M, Spertini F, et al. Api m 6: a new bee venom allergen. J Allergy Clin Immunol. (2001) 107:914-20. doi: $10.1067 /$ mai.2001.113867

213. Peiren N, de Graaf DC, Brunain M, Bridts CH, Ebo DG, Stevens WJ, et al. Molecular cloning and expression of icarapin, a novel IgE-binding bee venom protein. FEBS Lett. (2006) 580:4895-9. doi: 10.1016/j.febslet.2006.08.005

214. Li J, Wang T, Zhang Z, Pan Y. Proteomic analysis of royal jelly from three strains of western honeybees (Apis mellifera). J Agric Food Chem. (2007) 55:8411-22. doi: 10.1021/jf0717440

215. Zhang Q, Sun T, Tuo X, Li Y, Yang H, Deng J. A novel reversibly glycosylated polypeptide-2 of bee pollen from rape (Brassica napus L.): purification and characterization. Protein Peptide Lett. (2020) 28:543-53. doi: 10.2174/0929866527666201103161302

216. Buttstedt A, Moritz RF, Erler S. Origin and function of the major royal jelly proteins of the honeybee (Apis mellifera) as members of the yellow gene family. Biol Rev Cambridge Philos Soc. (2014) 89:255-69. doi: 10.1111/brv.12052

217. Shen LR, Wang YR, Zhai L, Zhou WX, Tan LL, Li ML, et al. Determination of royal jelly freshness by ELISA with a highly specific anti-apalbumin 1, major royal jelly protein 1 antibody. J Zhejiang Univ Sci B. (2015) 16:155-66. doi: $10.1631 /$ jzus.B1400223

218. Kamakura M. Royalactin induces queen differentiation in honeybees. Nature. (2011) 473:478-83. doi: 10.1038/nature10093

219. Kimura M, Kimura Y, Tsumura K, Okihara K, Sugimoto H, Yamada H, et al. $350-\mathrm{kDa}$ royal jelly glycoprotein (apisin), which stimulates proliferation of human monocytes, bears the betal-3galactosylated $\mathrm{N}$-glycan: analysis of the N-glycosylation site. Biosci Biotechnol Biochem. (2003) 67:2055-8. doi: 10.1271/bbb.67.2055

220. Kimura Y, Kajiyama S, Kanaeda J, Izukawa T, Yonekura M. N-linked sugar chain of 55-kDa royal jelly glycoprotein. Biosci Biotechnol Biochem. (1996) 60:2099-102. doi: 10.1271/bbb.60.2099
221. Kucharski R, Maleszka R. A royal jelly protein is expressed in a subset of kenyon cells in the mushroom bodies of the honey bee brain. Naturwissenschaften. (1998) 85:343-6. doi: 10.1007/s001140050512

222. Kader AA, Azmy R, Maher EA, El Sayed BB, Khalil AS, Ghalwash M, et al. Assessment of bee venom therapy in animal model of statininduced myopathy. Egyptian J Neurol Psychiatry Neurosurg. (2019) 55:71. doi: 10.1186/s41983-019-0120-9

223. Dacheux M, Sinou V, Payre C, Jeammet L, Parzy D, Grellier P, et al. Antimalarial activity of human group iia secreted phospholipase A2 in relation to enzymatic hydrolysis of oxidized lipoproteins. Infect Immunity. (2019) 87:e00556-19. doi: 10.1128/IAI.00556-19

224. Zahirovic A, Luzar J, Molek P, Kruljec N, Lunder M. Bee venom immunotherapy: current status and future directions. Clin Rev Allergy Immunol. (2020) 58:326-41. doi: 10.1007/s12016-019-08752-x

225. Lim HN, Baek SB, Jung HJ. Bee venom and its peptide component melittin suppress growth and migration of melanoma cells via inhibition of PI3K/AKT/mTOR and MAPK pathways. Molecules. (2019) 24:929. doi: 10.3390/molecules 24050929

226. Mohamed WA, Abd-Elhakim YM, Ismail SAA. Involvement of the antiinflammatory, anti-apoptotic, and anti-secretory activity of bee venom in its therapeutic effects on acetylsalicylic acid-induced gastric ulceration in rats. Toxicology. (2019) 419:11-23. doi: 10.1016/j.tox.2019.03.003

227. Marques Pereira AF, Albano M, Bergamo Alves FC, Murbach Teles Andrade BF, Furlanetto A, Mores Rall VL, et al. Influence of apitoxin and melittin from Apis mellifera bee on Staphylococcus aureus strains. Microbial Pathog. (2020) 141:104011. doi: 10.1016/j.micpath.2020.104011

228. Leandro LF, Mendes CA, Casemiro LA, Vinholis AH, Cunha WR, de Almeida R, et al. Antimicrobial activity of apitoxin, melittin and phospholipase A of honey bee (Apis mellifera) venom against oral pathogens. Anais Acad Brasileira Ciencias. (2015) 87:147-55. doi: 10.1590/0001-3765201520130511

229. Ham HJ, Han JH, Lee YS, Kim KC, Yun J, Kang SK, et al. Bee venom soluble phospholipase A2 exerts neuroprotective effects in a lipopolysaccharideinduced mouse model of alzheimer's disease via inhibition of nuclear factorkappa B. Front Aging Neurosci. (2019) 11:287. doi: 10.3389/fnagi.2019.00287

230. Boutrin MC, Foster HA, Pentreath VW. The effects of bee (Apis mellifera) venom phospholipase A2 on Trypanosoma brucei brucei and enterobacteria. Exp Parasitol. (2008) 119:246-51. doi: 10.1016/j.exppara.2008.02.002

231. Kim KH, Lee SY, Shin J, Hwang JT, Jeon HN, Bae H. Dose-Dependent neuroprotective effect of standardized bee venom phospholipase A2 against MPTP-induced Parkinson's disease in mice. Front Aging Neurosci. (2019) 11:80. doi: 10.3389/fnagi.2019.00080

232. Maqsoudlou A, Mahoonak AS, Mora L, Mohebodini H, Toldra F, Ghorbani M. Peptide identification in alcalase hydrolysated pollen and comparison of its bioactivity with royal jelly. Food Res Int. (2019) 116:905-15. doi: 10.1016/j.foodres.2018.09.027

233. Zhang X, Yu Y, Sun P, Fan Z, Zhang W, Feng C. Royal jelly peptides: potential inhibitors of beta-secretase in N2a/APP695swe cells. Sci Rep. (2019) 9:168. doi: 10.1038/s41598-018-35801-w

234. Bilikova K, Huang SC, Lin IP, Simuth J, Peng CC. Structure and antimicrobial activity relationship of royalisin, an antimicrobial peptide from royal jelly of Apis mellifera. Peptides. (2015) 68:190-6. doi: 10.1016/j.peptides.2015. 03.001

235. Guo H, Kouzuma Y, Yonekura M. Structures and properties of antioxidative peptides derived from royal jelly protein. Food Chem. (2009) 113:238-45. doi: 10.1016/j.foodchem.2008.06.081

236. Romanelli A, Moggio L, Montella RC, Campiglia P, Iannaccone M, Capuano F, et al. Peptides from Royal Jelly: studies on the antimicrobial activity of jelleins, jelleins analogs and synergy with temporins. J Peptide Sci. (2011) 17:348-52. doi: $10.1002 / \mathrm{psc} .1316$

237. Šimúth J. Some properties of the main protein of honeybee (Apis mellifera) royal jelly. Apidologie. (2001) 32:69-80. doi: 10.1051/apido:2001112

238. Klaudiny J, Hanes J, Kulifajová J, Albert Š, Šimúth J. Molecular cloning of two cDNAs from the head of the nurse honey bee (Apis mellifera $\mathrm{L}$.) for coding related proteins of royal jelly. J Apicultural Res. (2015) 33:105-11. doi: 10.1080/00218839.1994.11100857

239. Horio T, Kubo, Natori, S. Purification and cDNA cloning of the alcohol dehydrogenase of the flesh fly Sarcophaga peregrina - a structural 
relationship between alcohol dehydrogenase and a $25-\mathrm{kDa}$ protein. Euro J Biochem. (1996) 237:698-703. doi: 10.1111/j.1432-1033.1996.0698p.x

240. Albert S, Klaudiny J. The MRJP/YELLOW protein family of Apis mellifera: identification of new members in the EST library. J Insect Physiol. (2004) 50:51-9. doi: 10.1016/j.jinsphys.2003.09.008

241. Bellik Y. Bee Venom: Its Potential Use in Alternative Medicine. Anti-Infective Agents. (2015) 13:3-16. doi: 10.2174/2211352513666150318234624

242. Han SM, Park KK, Nicholls YM, Macfarlane N, Duncan G. Effects of honeybee (Apis mellifera) venom on keratinocyte migration in vitro. Pharmacognosy Magazine. (2013) 9:220-6. doi: 10.4103/0973-1296.113271

243. Memariani H, Memariani M. Anti-fungal properties and mechanisms of melittin. Appl Microbiol Biotechnol. (2020) 104:6513-26. doi: 10.1007/s00253-020-10701-0

244. Li L, Zhang S, Wei L, Wang Z, Ma W, Liu F, et al. Anti-fibrotic effect of melittin on TRIM47 expression in human embryonic lung fibroblast through regulating TRIM47 pathway. Life Sci. (2020) 256:117893. doi: $10.1016 /$ j.lfs.2020.117893

245. Choi S, Chae HK, Heo H, Hahm DH, Kim W, Kim SK. Analgesic effect of melittin on oxaliplatin-induced peripheral neuropathy in rats. Toxins. (2019) 11:396. doi: 10.3390/toxins11070396

246. Memariani H, Memariani M, Moravvej H, Shahidi-Dadras M. Melittin: a venom-derived peptide with promising anti-viral properties. Euro J Clin Microbiol Infect Dis. (2020) 39:5-17. doi: 10.1007/s10096-019-03674-0

247. Kurek-Gorecka A, Gorecki M, Rzepecka-Stojko A, Balwierz R, Stojko J. Bee products in dermatology and skin care. Molecules. (2020) 25:256. doi: 10.3390/molecules 25030556

248. Almeida JdF, Reis ASd, Heldt LFS, Pereira D, Bianchin M, Moura Cd, et al. Lyophilized bee pollen extract: a natural antioxidant source to prevent lipid oxidation in refrigerated sausages. LWT Food Sci Technol. (2017) 76:299305. doi: 10.1016/j.lwt.2016.06.017

249. Rzepecka-Stojko A, Stojko J, Kurek-Gorecka A, Gorecki M, KabalaDzik A, Kubina R, et al. Polyphenols from bee pollen: structure, absorption, metabolism and biological activity. Molecules. (2015) 20:2173249. doi: 10.3390/molecules 201219800

250. Kaškoniene V, Adaškevičiute $V$, Kaškonas $P$, Mickiene R, Maruška A. Antimicrobial and antioxidant activities of natural and fermented bee pollen. Food Biosci. (2020) 34:100532. doi: 10.1016/j.fbio.2020.100532

251. Sobral F, Calhelha RC, Barros L, Duenas M, Tomas A, SantosBuelga C, et al. Flavonoid composition and antitumor activity of bee bread collected in Northeast Portugal. Molecules. (2017) 22:248. doi: 10.3390/molecules 22020248

252. Rocchetti G, Castiglioni S, Maldarizzi G, Carloni P, Lucini L. UHPLCESI-QTOF-MS phenolic profiling and antioxidant capacity of bee pollen from different botanical origin. Int J Food Sci Technol. (2019) 54:335-46. doi: 10.1111/ijfs.13941

253. Kostić AŽ, Milinčić DD, Gašić UM, Nedić N, Stanojević SP, Tešić ŽL, et al. Polyphenolic profile and antioxidant properties of bee-collected pollen from sunflower (Helianthus annuus L.) plant. LWT. (2019) 112:108244. doi: 10.1016/j.lwt.2019.06.011

254. Emmanuel Atsalakisa IC, Maria Makropouloua, Sofia Karabourniotib and Konstantia Graikou. Evaluation of Phenolic Compounds in Cistus creticus Bee Pollen from Greece. Antioxidant and Antimicrobial Properties. Nat. Product Commun. (2017) 12:1813-6. doi: 10.1177/1934578X17012 01141

255. Su J, Yang X, Lu Q, Liu R. Antioxidant and anti-tyrosinase activities of bee pollen and identification of active components. J Apicultural Res. (2020) 60:297-307. doi: 10.1080/00218839.2020.1722356

256. Waś E, Szczesna T, Rybak-Chmielewska H, Teper D, Jaśkiewicz K. Application of HPLC-DAD technique for determination of phenolic compounds in bee pollen loads. J Apicultural Sci. (2017) 61:153-62. doi: 10.1515/jas-2017-0009

257. Giampieri F, Quiles JL, Orantes-Bermejo FJ, Gasparrini M, ForbesHernandez TY, Sanchez-Gonzalez C, et al. Are by-products from beeswax recycling process a new promising source of bioactive compounds with biomedical properties? Food Chem Toxicol. (2018) 112:126-33. doi: $10.1016 /$ j.fct.2017.12.041

258. NISbet C, Aker D. Antioxidant activities, total phenolic and flavonoid contents of honey collected from different botanical origins.
Ankara Üniversitesi Veteriner Fakültesi Dergisi. (2020) 67:133-6. doi: 10.33988/auvfd.523745

259. Mosic M, Trifkovic J, Vovk I, Gasic U, Tesic Z, Sikoparija B, et al. Phenolic composition influences the health-promoting potential of beepollen. Biomolecules. (2019) 9:783. doi: 10.3390/biom9120783

260. Bakour M, Al-Waili NS, El Menyiy N, Imtara H, Figuira AC, Al-Waili $\mathrm{T}$, et al. Antioxidant activity and protective effect of bee bread (honey and pollen) in aluminum-induced anemia, elevation of inflammatory makers and hepato-renal toxicity. J Food Sci Technol. (2017) 54:4205-12. doi: 10.1007/s13197-017-2889-9

261. Mushtaq M, Lateef A, Ahmad H, Bono A, Nisar A. Simultaneous identification of phenolic compound from the honey of stingless bee by using HPLC. Recent Adv Biol Med. (2019) 5:1. doi: 10.18639/RABM.2019.9 61432

262. Urcan AC, Criste AD, Dezmirean DS, Margaoan R, Caeiro A, Graca Campos M. Similarity of data from bee bread with the same taxa collected in India and Romania. Molecules. (2018) 23:2491. doi: 10.3390/molecules23102491

263. de Souza SA, da Silva TMG, da Silva EMS, Camara CA, Silva TMS. Characterisation of phenolic compounds by UPLC-QTOF-MS/MS of geopropolis from the stingless bee Melipona subnitida (jandaira). Phytochem Anal PCA. (2018) 29:549-58. doi: 10.1002/pca.2766

264. Al-Hatamleh MAI, Boer JC, Wilson KL, Plebanski M, Mohamud R, Mustafa MZ. Antioxidant-based medicinal properties of stingless bee products: recent progress and future directions. Biomolecules. (2020) 10:293. doi: 10.3390/biom10060923

265. Ghouizi AE, Menyiy NE, Falcao SI, Vilas-Boas M, Lyoussi B. Chemical composition, antioxidant activity, and diuretic effect of Moroccan fresh bee pollen in rats. Vet World. (2020) 13:1251-61. doi: 10.14202/vetworld.2020.1251-1261

266. Thakur M, Nanda V. Screening of Indian bee pollen based on antioxidant properties and polyphenolic composition using UHPLC-DAD-MS/MS: a multivariate analysis and ANN based approach. Food Res Int. (2021) 140:110041. doi: 10.1016/j.foodres.2020.110041

267. Perveen S, Orfali R, Al-Taweel AM, Khan A, Alghanem B, Shaibah H. Simultaneous identification of phenolic and flavonoid contents in bee pollen by HPLC-ESI-MS data. Biomed Res. (2019) 30:770-4. doi: 10.35841/biomedicalresearch.30-19-278

268. Aylanc V, Falcão SI, Ertosun S, Vilas-Boas M. From the hive to the table: nutrition value, digestibility and bioavailability of the dietary phytochemicals present in the bee pollen and bee bread. Trends Food Sci Technol. (2021) 109:464-81. doi: 10.1016/j.tifs.2021.01.042

269. Keskin S, Trusheva B, Keskin M, Popova M, Kolayli S, Bankova V. Pollen beads: a new carrier for propolis active compounds. Combinatorial Chem High Throughput Screening. (2020) 24:1688-95. doi: 10.2174/1386207323666200720102651

270. Yang YF, Lai XY, Lai GY, Jiang ZD, Ni H, Chen F. Purification and characterization of a tyrosinase inhibitor from camellia pollen. J Funct Foods. (2016) 27:140-9. doi: 10.1016/j.jff.2016.08.056

271. Yang YF, Lai XY, Huang GL, Chen YH, Du XP, Jiang ZD, et al. Isolation and purification of two tyrosinase inhibitors from camellia pollen by high-speed counter-current chromatography. Acta Chromatographica. (2017) 29:47783. doi: 10.1556/1326.2017.29407

272. Yang Y, Sun X, Ni H, Du X, Chen F, Jiang Z, et al. Identification and characterization of the tyrosinase inhibitory activity of caffeine from camellia pollen. J Agric Food Chem. (2019) 67:12741-51. doi: 10.1021/acs.jafc.9b04929

273. Cebi N, Bozkurt F, Yilmaz MT, Sagdic O. An evaluation of FTIR spectroscopy for prediction of royal jelly content in hive products. J Apicultural Res. (2020) 59:146-55. doi: 10.1080/00218839.2019.1707009

274. Vykydalová A, Cibulková Z, Cížová K, Vizárová K, Šimon P. Degradation of beeswax by NOx pollution and UV light studied by DSC and FTIR measurements. Thermochimica Acta. (2020) 689:178606. doi: 10.1016/j.tca.2020.178606

275. Alimoglu G, Guzelmeric E, Yuksel PI, Celik C, Deniz I, Yesilada E. Monofloral and polyfloral bee pollens: comparative evaluation of their phenolics and bioactivity profiles. LWT. (2021) 142:110973. doi: 10.1016/j.lwt.2021.110973

276. Thakur M, Nanda V. Composition and functionality of bee pollen: a review. Trends Food Sci Technol. (2020) 98:82-106. doi: 10.1016/j.tifs.2020.02.001 
277. Boselli E, Caboni MF, Sabatini AG, Marcazzan GL, Lercker G. Determination and changes of free amino acids in royal jelly during storage. Apidologie. (2003) 34:129-37. doi: 10.1051/apido:2003011

278. Xu X-l, Zheng Y-z, Chen X-c, Zhu F-l, Miao X-q. Identification of cattail pollen, pine pollen and bee pollen by Fourier transform infrared spectroscopy and two-dimensional correlation infrared spectroscopy. J Mol Struct. (2018) 1167:78-81. doi: 10.1016/j.molstruc.2018.04.076

279. Miłek M, Drogoń A, Pyda M, Czerniecka-Kubicka A, Tomczyk M, Dzugan M. The use of infrared spectroscopy and thermal analysis for the quick detection of adulterated beeswax. J Apicultural Res. (2020) 59:677-84. doi: 10.1080/00218839.2020.1773037

280. Kafantaris I, Amoutzias GD, Mossialos D. Foodomics in bee product research: a systematic literature review. Euro Food Res Technol. (2020) 247:309-31. doi: 10.1007/s00217-020-03634-5

281. Peres AM, Sousa MEB, Veloso ACA, Estevinho LM, Dias LG. Electrochemical sensors for assessing antioxidant capacity of bee product. In: Cardoso SM, Silva AMS, editors. Chemistry, Biology and Potential Applications of Honeybee Plant-Derived Products. Bentham Science Press (2016). p. 196-223. doi: 10.2174/9781681082370116010009

282. Mašková Z, Knazovická V, Mančíková V, Tančinová D, Barboráková Z. Monitoring of microscopic fungi community in selected bee products. Potravinarstvo Slovak J Food Sci. (2020) 14:1105-14. doi: 10.5219/ 1405

283. Campos MGR BS, Almeida-Muradian LB, Szczesna YM, Frigerio C, Ferreira F. Pollen composition and standardisation of analytical methods. J Apicultural Res and Bee World. (2008) 47:156-63. doi: 10.1080/00218839.2008.11101443

284. Serra J, Gómez A, Gonnell J. Composición, propiedades físico-químicas y espectro polínico de algunas mieles uniflorales de Espaa. Alimentaria Revista De Tecnología E Higiene De Los Alimentos. (1987) 185:61-84. Available online at: https://dialnet.unirioja.es/servlet/articulo? codigo $=5255665$

285. Yook HS, Lim SI, Byun MW. Changes in microbiological and physicochemical properties of bee pollen by application of gamma irradiation and ozone treatment. J Food Protection. (1998) 61:217-20. doi: 10.4315/0362-028X-61.2.217

286. Conti ME, Botrè F. Honeybeesand their products as potential bioindicators of heavymetals contamination. Environ Monitor Assessment. (2001) 69:26782. doi: 10.1023/A:1010719107006

287. Kubik M, Nowacki J, Pidek A, Warakomska Z, Michalczuk L, Goszczyñski W. Pesticide residues in bee products collected from cherry trees protected during blooming period with contact and systemic fungicides. Apidologie. (1999) 30:521-32. doi: 10.1051/apido:19990607

288. Tong Z, Duan J, Wu Y, Liu Q, He Q, Shi Y, et al. A survey of multiple pesticide residues in pollen and beebread collected in China. Sci Total Environ. (2018) 640-1:1578-86. doi: 10.1016/j.scitotenv.2018.04.424
289. Medina A, Gonzalez G, Saez JM, Mateo R, Jimenez M. Bee pollen, a substrate that stimulates ochratoxin A production by Aspergillus ochraceus Wilh. Systematic Appl Microbiol. (2004) 27:261-7. doi: 10.1078/072320204322881880

290. Manning R. Chemical residues in beebread, honey, pollen and wax samples collected from bee hives placed on canola crops in Western Australia. $J$ Apicultural Res. (2018) 57:696-708. doi: 10.1080/00218839.2018.1494889

291. Pietta PG, Gardana C, Pietta AM. Analytical methods for quality control of propolis. Fitoterapia. (2002) 73(Suppl. 1):S7-20. doi: 10.1016/S0367-326X(02)00186-7

292. Pellati F. Innovative methods for the extraction and chromatographic analysis of honey bee products. ACS Sym Ser. (2014) 1185:33-49. doi: 10.1021/bk-2014-1185.ch002

293. Li S, Shan Y, Zhu X, Zhang X, Ling G. Detection of honey adulteration by high fructose corn syrup and maltose syrup using Raman spectroscopy. $J$ Food Composition Anal. (2012) 28:69-74. doi: 10.1016/j.jfca.2012.07.006

294. Reybroeck W. Quality control of honey and bee products. In: Gupta RK, Reybroeck W, van Veen JW, Gupta A, editors. Beekeeping for Poverty Alleviation and Livelihood Security. Springer Press (2014) p.481-506. doi: 10.1007/978-94-017-9199-1_18

295. Božiková M, Hlaváč P, Vozárová V, Hlaváčová Z, Kubík L, Kotoulek P, et al. Thermal properties of selected bee products. Progress Agric Eng Sci. (2018) 14:37-44. doi: 10.1556/446.14.2018.s1.4

296. Bogdanov S, Ruoff K, Persano Oddo L. Physico-chemical methods for the characterisation of unifloral honeys: a review. Apidologie. (2004) 35(Suppl. 1):S4-17. doi: 10.1051/apido:2004047

Conflict of Interest: The authors declare that the research was conducted in the absence of any commercial or financial relationships that could be construed as a potential conflict of interest.

Publisher's Note: All claims expressed in this article are solely those of the authors and do not necessarily represent those of their affiliated organizations, or those of the publisher, the editors and the reviewers. Any product that may be evaluated in this article, or claim that may be made by its manufacturer, is not guaranteed or endorsed by the publisher.

Copyright (c) 2021 Luo, Dong, Gu, Zhang and Ma. This is an open-access article distributed under the terms of the Creative Commons Attribution License (CC BY). The use, distribution or reproduction in other forums is permitted, provided the original author(s) and the copyright owner(s) are credited and that the original publication in this journal is cited, in accordance with accepted academic practice. No use, distribution or reproduction is permitted which does not comply with these terms. 